Arthur Tarso Rego

\title{
Non-Gaussian Stochastic Volatility Model with Jumps via Gibbs Sampler
}

Modelo Não-Gaussiano de Volatilidade Estocástica com Saltos via

Amostrador de Gibbs

Belo Horizonte

2017 


\section{UNIVERSIDADE FEDERAL DE MINAS GERAIS \\ DEPARTAMENTO DE ESTATÍSTICA \\ PROGRAMA DE PÓS-GRADUAÇÃO EM ESTATÍSTICA}

Arthur Tarso Rego

\section{Non-Gaussian Stochastic Volatility Model with Jumps via Gibbs Sampler}

Modelo Não-Gaussiano de Volatilidade Estocástica com Saltos via Amostrador de Gibbs

Orientador: Prof. Thiago Rezende dos Santos

Belo Horizonte

2017 


\section{Resumo}

Compreender o comportamento dos ativos financeiros é de extrema importância para determinar a alocação de capital entre as diferentes formas de investimento disponíveis. Tal escolha depende, dentre outros fatores, da percepção do indivíduo acerca dos riscos e retornos associados a essas opções de investimentos. Na literatura, encontram-se diversos modelos cujo objetivo é estimar o risco de aplicações financeiras, entretanto, a maioria depende de métodos MCMC baseados em algoritmos Metropolis, o que os torna computacionalmente custosos. O presente trabalho apresenta um modelo alternativo, o Modelo Não Gassiano de Volatilidade Estocástica Com Saltos (NGSVJ), baseado nos Modelos Dinâmicos Lineares (DLM) com mistura, capaz de estimar a volatilidade sem recorrer a métodos computacionais intensivos, utilizando apenas o Amostrador de Gibbs. Isso é possível devido à estrutura do modelo, que permite obter as condicionais completas a posteriori para os parâmetros. Além disso, a inserção de saltos nos retornos no modelo o permite capturar os movimentos especulativos pontuais do mercado, sem que isso se traduza em aumento da volatilidade. São apresentados estudos de simulação a fim de investigar a eficácia do método proposto e, ao final, realiza-se uma análise com dados reais de retornos dos índices S\&P 500 e iBovespa. Os resultados indicam que o modelo proposto foi capaz de estimar a volatilidade, com resultados semelhantes aos de outros modelos existentes na literatura, de forma computacionalmente eficaz e automatizada, sendo que sua capacidade de identificar saltos é maior quando a série de retornos em estudo possui movimentos especulativos fortes. Um método não paramétrico também foi utilizado para estimar os saltos, mas os resultados de simulação mostraram que ele não foi eficaz.

Palavras-chave: Séries Temporais, Modelos de Volatilidade Estocástica, Amostrador de Gibbs, Modelos Lineares Dinâmicos, Cauda Pesada, Inferência Bayesiana. 


\section{Abstract}

To understand the behavior of asset prices is essential for capital allocation decisions between the available investment option. Such decision depends on what one thinks about risks and returns associated with these investment options. On the literature there are models focused on estimating financial assets risk, however, most of them depend on MCMC methods based on Metropolis algorithms, which makes them computationally expensive. This work presents an alternative model, the Non Gaussian Stochastic Volatility Model with Jumps (NGSVJ), based on the Dynamic Linear Model (DLM) with mixture, capable of estimating the volatility without appealing to intensive computational methods, using only Gibbs Sampler. This is possible due to the model structure, which allows the posterior full conditionals for parameters. Besides that, the insertion of jumps on modeling the returns allows it to capture isolated speculative movements of the market, without transferring its effects to volatility. Simulated studies are presented in order to investigate the efficiency of the proposed method. Finally, an analysis with real data is performed using return series from S\&P 500 and iBovespa indexes. Results indicate that the proposed model was capable of estimating volatility, with results similar to other models on literature, in a computationally efficient and automated way, being that its ability to identify jumps is greater when the series of returns under study has strong speculative movements. A non-parametric method was also used for estimating jumps, but simulation results showed that it was not effective.

Key-words: Time Series, Stochastic Volatility Models, Gibbs Sampler. Dynamic Linear Models, Heavy Tails, Bayesian Inference. 


\section{List of Figures}

3.1 Normal Simulations - B-statistic comparison . . . . . . . . . . . . . 29

3.2 Simulation 1 - Normal with Strong Jumps - Jump probabilities and

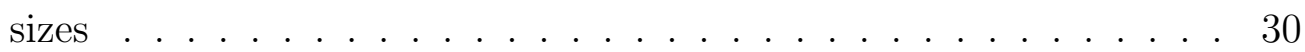

3.3 Simulation 2 - Normal with Weak Jumps - Jump probabilities and sizes . . . . . . . . . . . . . . . . . . 31

3.4 Simulation 3 - Normal with Manually Imputed Jumps - Jump probabilities and sizes . . . . . . . . . . . . . . . . 32

3.5 B-statistics comparison for Student's t scenario . . . . . . . . . . 35

3.6 Simulation 4 - Strong Jumps - Jump probabilities and sizes . . . . . 36

3.7 Simulation 4 - Weak Jumps - Jump probabilities and sizes . . . . . 37

3.8 Simulation 4 - Manually Imputed Jumps - Jump probabilities and sizes . . . . . . . . . . . . . . . . . . . . 38

3.9 Simulation 5 - Influence of volatility changes . . . . . . . . . . . . 39

4.1 S\&P500 log return histogram . . . . . . . . . . . . . . . 42

4.2 iBovespa log return histogram . . . . . . . . . . . . . . . . . . . . 42

$4.3 \mathrm{~S} \& \mathrm{P} 500 \log$ return series . . . . . . . . . . . . . . . . . 42

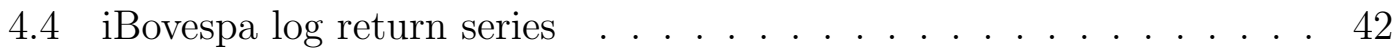

4.5 Estimated jump times and sizes for the S\&P500 index . . . . . . . 45 
4.6 Estimated volatility for the S\&P500 index. Continuous line represents the posterior mean. The grey area indicates the $95 \%$ credibility intervals. . . . . . . . . . . . . . . . . 4 46

4.7 Mixture component, $\gamma_{t} \ldots \ldots \ldots \ldots$. . . . . . . . . . 46

4.8 Variance, $\gamma_{t}^{-1} \lambda_{t}^{-1} \ldots \ldots \ldots \ldots \ldots$. . . . . . . . . . 46

4.9 Estimated jump times and sizes for the iBovespa index . . . . . . . 49

4.10 Estimated volatility for the iBovespa index. Continuous line represents the posterior mean. The grey area indicates the $95 \%$ credibility intervals. . . . . . . . . . . . . . . . 5 50

4.11 Mixture component, $\gamma_{t} \ldots \ldots \ldots$. . . . . . . . . . . 50

4.12 Variance, $\gamma_{t}^{-1} \lambda_{t}^{-1} \ldots \ldots \ldots \ldots \ldots$. . . . . . . . . . . 50

5.1 Block average jump detection flowchart. Source: Riley(2008). . . . . 53

5.2 B-statistics comparison for Student's t scenario - Non Parametric Approach . . . . . . . . . . . . . . . . . 5 54

5.3 Simulation 6 - Weak Jumps - Non-Parametric Approach . . . . . . 57

6.1 Comparison between estimated volatility on NGSVJ model and Eraker et al. (2003) [9] models. . . . . . . . . . . . . . . . . . . 61

D.1 MCMC results for S\&P 500 log returns data. . . . . . . . . . 76

D.2 MCMC results for iBovespa log returns data. . . . . . . . . . . . 77 


\section{List of Tables}

3.1 Manually imputed jumps . . . . . . . . . . . . . . . . 23

3.2 Summary of Simulation Scenarios . . . . . . . . . . . . . . . 24

3.3 Simulation 1 - Normal with Strong Jumps . . . . . . . . . . . 26

3.4 Simulation 2 - Normal with Weak Jumps . . . . . . . . . . . . . . 27

3.5 Simulation 3 - Normal with Manually Imputed Jumps . . . . . . . . 28

3.6 Simulation 4 - Student's t - 20 degrees of freedom . . . . . . . . . 34

4.1 Summary Statistics . . . . . . . . . . . . . . . . . . 42

4.2 Parameter estimates for S\&P 500 index data . . . . . . . . . . . . 43

4.3 Parameter estimates for iBovespa index data . . . . . . . . . . . . 47

5.1 Simulation 6 - Non-Parametric Approach - Student's t - 20 degrees of freedom . . . . . . . . . . . . . . . . . . 55

6.1 Comparison between NGSVJ model and SVJ models in Eraker et. al (2003) [9] for S\&P 500 returns . . . . . . . . . . . . . 60 


\section{Contents}

$\begin{array}{llr}1 & \text { Introduction } & 9\end{array}$

1.1 Some models on literature . . . . . . . . . . . . . . . . . 10

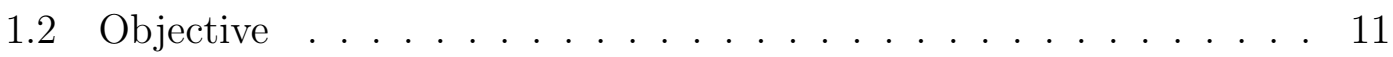

2 The proposed model 13

2.1 DLM Model with Scale Mixtures . . . . . . . . . . . . . . . 13

2.2 Stochastic Volatility Model with Jumps . . . . . . . . . . . . . . . . 15

2.3 Non-Gaussian Stochastic Volatility Model with Jumps on Returns . 16

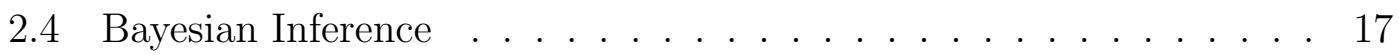

2.4.1 NGSVJ related parameters: conditional posteriors . . . . . . 17

2.4.2 Jump related parameters . . . . . . . . . . . . . . . . . 18

2.4.3 MCMC Algorithm . . . . . . . . . . . . . 20

2.4.4 Model Diagnostics and Specification Tests . . . . . . . . . . 21

3 Simulations $\quad 22$

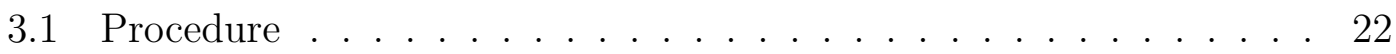

3.2 Results and comments . . . . . . . . . . . . . . . 24

3.2.1 Simulations for the Gaussian approach . . . . . . . . . . 25

3.2.2 Sudent's t Simulations . . . . . . . . . . . . . . . 32 
3.3 Simulation conclusions . . . . . . . . . . . . . . . . 40

4 Applying model to stock market index data 41

$4.1 \quad \mathrm{~S} \& \mathrm{P} 500 \ldots \ldots \ldots \ldots \ldots \ldots$

4.2 iBovespa . . . . . . . . . . . . . . . . . . . . . 47

4.3 Experimental conclusions . . . . . . . . . . . . . . . 51

5 A proposal for weak jumps scenario $\quad 52$

5.1 Jump Detection Algorithm: a Non-Parametric Approach . . . . . . 52

5.2 Simulations ............................ 54

5.2 .1 Simulation conclusions . . . . . . . . . . . . 57

6 Discussion about the NGSVJ model $\quad 58$

6.1 NGSVJ model advantages . . . . . . . . . . . . . . . . 58

6.2 Comparison to other models . . . . . . . . . . . . . . 60

$\begin{array}{lll}7 & \text { Conclusion } & 62\end{array}$

A Full Conditional Posterior Distributions for NGSV Model $\quad 67$

A.1 Conditional Posterior Distribution for $\gamma_{t} \ldots \ldots$. . . . . . . 67

A.2 Conditional Posterior Distribution for $\delta_{t} \ldots \ldots \ldots$. . . . . . . 68

A.3 Conditional Posterior Distribution for $\sigma_{\mu}^{2} \ldots \ldots$. . . . . . . . 68

B Full Conditional Posterior Distributions for Jump Components 69

B.1 Conditional Posterior Distribution for $\mu_{y} \ldots \ldots . . . . . . .70$

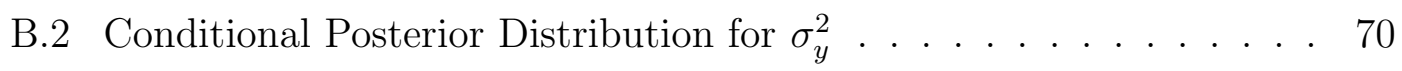

B.3 Conditional Posterior Distribution for $\xi_{t+1}^{y} \ldots \ldots \ldots 71$

B.4 Conditional Posterior Distribution for $\rho \ldots \ldots . . . . . . . .71$

B.5 Conditional Posterior Distribution for $N_{t+1}^{y} \ldots \ldots . \ldots 71$ 
C Sampling from $\lambda_{t}$

$\begin{array}{ll}\text { D MCMC Chains for NGSVJ Model } & 75\end{array}$

D.1 S\&P 500 MCMC results . . . . . . . . . . . . . . 76

D.2 iBovespa MCMC results . . . . . . . . . . . . . . 77 


\section{Chapter 1}

\section{Introduction}

"The behavior of asset prices is essential for many important decisions, not only for professional investors but also for most people in their daily life. The choice between saving in the form of cash, bank deposits or stocks, or perhaps a single-family house, depends on what one thinks of the risks and returns associated with these different forms of saving." (Kungl. Vetenskaps-Akademien, 2013) [13]. This field attracts researches trying to figure out the main market drivers and how asset returns are influenced by them. The most accepted theory is that the returns on high volatility ${ }^{1}$ assets follow a random walk with some outliers, that usually occur during abnormal volatility increases, such as in financial and political crisis events. The future returns would be unpredictable, but the volatility can be estimated and monitored in order to detect the approach of such events and antecipate its movements.

The stochastic volatility models commonly used are not much efficient on dealing with high-dimensional data, since Bayesian inference is mostly based on Markov Chain Monte Carlo (MCMC) methods, for example, using MetropolisHastings algorithms, which raises questions about the usage of more efficient methods that can be used to bring tangible results in a shorter period of time.

\footnotetext{
${ }^{1}$ Measurement of assets return dispersion, variance. The higher an asset price changes in a short period of time, higher is the risk of earnings or losses on trading this asset. Thus, volatility can be seen as a risk measurement of a financial asset.
} 
Dealing with financial time series brings three main challenges. They include finding a model that: fits well to the data, accommodating the heavy tails that exist in non-Gaussian returns; is fast enough to bring results on time to be used by market agents; is flexible to include new source of data and accommodates outliers and skewness, that improve the model performance.

\subsection{Some models on literature}

A simple model designed to describe high volatility assets behavior was developed by Schwartz and Smith (2000) [22]. Their model consists of decomposing the asset log returns in the short-term deviation in prices and the equilibrium price. To estimate their values, a Kalman Filter is used, assuming, therefore, a Gaussian distribution for the errors. The future contract prices, which in economic theory is believed to represent the market expectations about the convergence of asset prices in the future, are used to estimate the equilibrium price. The parameter estimates were obtained by the authors numerically, using the "maxlik" (Maximum Likelihood) routine in econometric software GAUSS. This model, however, does not work well to day-by-day applications since it is not able to adjust to moments with high volatility in market. It also simplifies too much the reality on considering Gaussian distribution to returns.

Eraker, Johannes e Polson (2003) [9] adopt the Stochastic Volatility model (SV) and study the influence of inserting jumps to improve the model. They suggest including jumps on returns and volatility in order to improve the model dynamic in case of spot changes in volatility, as in financial crisis moments. The main contribution of their work was allowing to understand the influence of Jumps in the model capability of reflecting speculative movements on financial markets. The advantage of adopting this model is that it fits better to the market movements, with good results for risk analysis and option pricing. On the other hand, the parameter estimation is somewhat complex, since there are no closed form to the full conditional distributions, being necessary the use of MCMC methods with Metropolis steps, which is less efficient in case of high-dimensional data. Other 
point is that this model still simplifies reality by assuming Gaussian distribution for errors, underestimating the effect of heavy tails. It also does not allow the inclusion of exogenous variables, such as future contract prices.

Other models such as the one developed by Warty, Lopes and Polson (2014) [26] brings some innovations by using State Space Models (SSM) and sequential MCMC methods in order to model the returns, allowing the introduction of Jumps and correlated variables, but still assuming Gaussian errors. Brooks and Prokopczuk (2011) [3] extend the Stochastic Volatility with Jumps (SVJ) model to an asset portfolio - the multivariate case, which is closer to the day-by-day reality. Omori et. al (2006) [18] include the leverage effect on SV models, which refers to the increase in volatility following a previous drop in stock returns, and modeled by the negative correlation coefficient between error terms of stock returns. Nakajima and Omori (2007) [17] extend its application to SVJ models with heavy-tail distribution obtained by a scale mixture of a generalized gamma distributed mixture component together with a normal distributed error in order to generate generalized Skew-t distributed innovations, discussing the fitness gains on including such feature. Merener (2015) [16] uses a GARCH model including jumps in returns and volatility, including exogenous variables in the model based on Supply and Demand concept ${ }^{2}$, with innovations assuming a Student-t distribution to better accommodate financial data.

\subsection{Objective}

Since there are a lot of works on stochastic volatility models for univariate financial time series, so, one might ask what is the gain on continue developing methods to model volatility for univariate financial time series. Such models are not very efficient on dealing with high dimensional data, since they rely on computationally intensive methods, such MCMC with Metropolis steps, which have

\footnotetext{
${ }^{2}$ Supply shocks would affect the demand for specific commodities or assets and result in extreme returns, hence the inclusion of co-variables related to the supply and demand quantity on trying to explain returns.
} 
several tunning parameters, and have a complex structure.

With the discussion about financial time series models on background, the main goal of this work is finding an alternative model that accommodates speculative financial asset returns data, allowing the innovations to assume heavy tails distributions; include jumps on returns in order to get the impact of uncommon events on financial markets; find a faster computational procedure, like Gibbs Sampling together with a block sampling structure, to estimate parameters, without appealing to intensive computational resources, such as Metropolis steps for each parameter.

This work will be divided in two parts. In the first part the proposed model will be detailed, in Chapter 2, as well as simulation studies, Chapter 3, and its results on estimating volatility for the S\&P-500 and iBovespa indexes, Chapter 4. In the second part there will be a discussion about the use of an alternative method to detect jump times for weak jumps scenario, using a non-parametric approach,Chapter 5 and how it affects the model.

The main contributions of this work are: introducing an automatic DLM model with jumps and heavy tailed distribution to be applied financial assets return data, using Gibbs Sampler; presenting simulation studies that attest its efficiency on estimating volatility and capturing speculative movements, represented by jumps; applying the model to real financial return time series and presenting its results. This work proposes a model that is computationally efficient and automatic in order to estimate volatility for financial return time series, that can be run on an ordinary home computer and give reliable results for analysis in a little time for high dimensional data, so that it can be used by market players in order to build investment strategies. 


\section{Chapter 2}

\section{The proposed model}

This chapter presents the proposed model and its properties. This approach uses only returns data to estimate the models, so that its results are comparable to the model proposed by Eraker et al. (2003) [9], although it can be extended to include any other exogenous variable that may be relevant on the analysis, as can be seen on Gamerman, Santos and Franco (2013) [10].

\subsection{DLM Model with Scale Mixtures}

The Dynamic Linear Model (DLM) with scale mixtures was proposed by Gamerman et al. (2013) [10] and is a extension of the classic DLM, which can be found on West and Harrison (1997) [27]. This model provides the necessary flexibility by using the SSM form, with mixtures on variance in order to achieve non-Gaussian distribution for innovations. It has also a formulation that allows the full conditional distributions to be available, so that a Gibbs Sampler can be used to sample from the full conditional posterior distributions, decreasing the computational time and bringing implementation simplicity to the model.

The DLM with scale mixtures (Gamerman et al., 2013) [10] can be written 
as:

$$
\begin{aligned}
y_{t} & =F_{t} \theta_{t}+v_{t}, \quad \text { where } v_{t} \mid \gamma_{t} \sim N\left(0, \gamma_{t}^{-1} \lambda_{t}^{-1}\right), \\
\theta_{t} & =G_{t-1} \theta_{t-1}+\omega_{t}, \quad \text { where } \omega_{t} \mid \delta_{t}, \varphi \sim N\left(0, \delta_{t}^{-1} W\right), \\
\lambda_{t} & =\omega^{-1} \lambda_{t-1} \zeta_{t}, \quad \text { where } \zeta_{t} \mid Y_{t-1}, \varphi \sim \operatorname{Beta}\left(\omega a_{t-1},(1-\omega) a_{t-1}\right), \\
\theta_{0} \mid Y_{0} & \sim N\left(m_{0}, C_{0}\right) \quad \text { independent of } \lambda_{0} \mid Y_{0} \sim \operatorname{Gamma}\left(a_{0}, b_{0}\right) .
\end{aligned}
$$

In this model, $y_{t}$ denotes the $t^{t h}$ value of the series, $F_{t}$ and $G_{t}$ are system matrices, $\theta_{t}$ is the vector of latent states, $\lambda_{t}$ is the precision, always positive, hence $\lambda_{t}^{-1}$ is the volatility, $Y_{t}$ is observed value of the series on time t, $\varphi=(\omega, \operatorname{diag}(W))$ is the model hyperparameter and $a_{t-1}$ is a form parameter of the filtering distribution for $\lambda_{t}$.

This approach allows inserting exogenous variables directly on returns through the $F_{t}$ vector. Also, heavy tail distributions for innovations, such as Student-t, logistic and GED, can be obtained through the scale mixture on determining $\gamma_{t}$ and $\delta_{t}$. For example, if $\gamma_{t}$ and $\delta_{t} \sim \operatorname{Gamma}\left(\frac{\nu}{2}, \frac{\nu}{2}\right)$, then unconditional errors would assume a $t_{\nu}\left(0, \lambda_{t}^{-1}\right)$ distribution. If their values is fixed as 1 , than unconditional errors would assume a Normal distribution.

In this case, there are no dimensionality issues with the parametric space, since all complete conditional distributions are obtained through the model properties and sampling from the marginal posterior can be made through a Gibbs Sampler algorithm. Using proper priors to $\operatorname{diag}(\mathrm{W})$ makes it possible to obtain the posterior complete conditionals, where the priors are chosen in order to result in a proper posterior distribution. This procedure consists on block-sampling: first the static and then dynamic parameters. Such procedure leads to a much faster estimation, since there is no need to use Metropolis-Hasting algorithms, as on other models exposed previously.

Another advantage lays on sampling mean components $\theta_{0: n}$ and volatility $\lambda_{0: n}$ in blocks, mitigating convergence problems. A more detailed description for the model procedures can be seen in Gamerman et al. (2013) [10]. 


\subsection{Stochastic Volatility Model with Jumps}

Eraker et al. (2003) [9] adopt the Stochastic Volatility (SV) model and study the influence of inserting jumps to improve the model. The structure proposed by them gives an insight on how to insert jumps in DLM with mixtures on scale. In their model, jumps are inserted in an additive form in both, returns and volatility.

In the model proposed by Eraker et al. (2003) [9], the log price of an asset $y_{t}=\log \left(S_{t}\right)$, where $S_{t}$ is the asset price on time $\mathrm{t}$, is given by:

$$
\begin{aligned}
& y_{t+1}=y_{t}+\mu+\sqrt{v_{t} \epsilon_{t+1}^{y}}+J_{t+1}^{y} \\
& v_{t+1}=v_{t}+\kappa\left(\theta-v_{t}\right)+\sigma_{v} \sqrt{v_{t} \epsilon_{t+1}^{v}}+J_{t+1}^{v}
\end{aligned}
$$

In which $\epsilon_{t+1}^{y}$ and $\epsilon_{t+1}^{v}$ are $N(0,1)$ with $\operatorname{corr}\left(\epsilon_{t+1}^{y}, \epsilon_{t+1}^{v}\right)=\rho$, between -1 and 1 , and the jump components are:

$$
J_{t}^{y}=\xi_{t+1}^{y} N_{t+1}^{y}, \quad J_{t}^{v}=\xi_{t+1}^{v} N_{t+1}^{v}, \quad \operatorname{Pr}\left(N_{t+1}^{y}=1\right)=\rho_{y}, \quad \operatorname{Pr}\left(N_{t+1}^{v}=1\right)=\rho_{v} .
$$

The jumps, therefore, are composed by a component that denotes the presence of a jump at instant $\mathrm{t}+1$, represented by $N_{t+1}^{y}$ and $N_{t+1}^{v}$, which can assume only two values: 1 or 0 ; and a component that denotes the jump magnitude at the same time, $\xi_{t+1}^{y} \sim N\left(\mu_{y}, \sigma_{y}^{2}\right)$ and $\xi_{t+1}^{v} \sim \exp \left(\mu_{v}\right)$.

This method of including jumps can be adapted to the DLM with mixture on scales to get advantage of its properties that guarantee a faster sampling method, through block sampling, and more efficient due to Gibbs Sampling. For this work the concern is about including jumps only in returns, since including them also on volatility requires a more complex structure in order to preserve the model properties and a fast inference procedure, and will be addressed in future works. 


\subsection{Non-Gaussian Stochastic Volatility Model with Jumps on Returns}

The Non-Gaussian Stochastic Volatility Model with jumps on returns (NGSVJ) can be written as:

$$
\begin{aligned}
y_{t} & =F_{t} \theta_{t}+J_{t}^{y}+v_{t}, \quad \text { where } v_{t} \mid \gamma_{t} \sim N\left(0, \gamma_{t}^{-1} \lambda_{t}^{-1}\right), \\
\theta_{t} & =G_{t} \theta_{t-1}+\omega_{t}, \quad \text { where } \omega_{t} \mid \delta_{t}, \varphi \sim N\left(0, \delta_{t}^{-1} \sigma_{\mu}^{2}\right), \\
\lambda_{t} & =\omega^{-1} \lambda_{t-1} \zeta_{t}, \quad \text { where } \zeta_{t} \mid Y_{t-1}, \varphi \sim \operatorname{Beta}\left(\omega a_{t-1},(1-\omega) a_{t-1}\right), \\
\mu_{0} \mid Y_{0} & \sim N\left(m_{0}, C_{0}\right) \quad \text { independent of } \lambda_{0} \mid Y_{0} \sim \operatorname{Gamma}\left(a_{0}, b_{0}\right) .
\end{aligned}
$$

where:

$$
J_{t}^{y}=\xi_{t+1}^{y} N_{t+1}^{y}, \quad \xi_{t+1}^{y} \sim N\left(\mu_{y}, \sigma_{y}^{2}\right), \quad \text { and } \quad \operatorname{Pr}\left(N_{t+1}^{y}=1\right)=\rho_{y} .
$$

In this model, $y_{t}$ represents the log-return in percentage, defined as $y_{t}=$ $100 *\left(\log \left(S_{t}\right)-\log \left(S_{t-1}\right)\right)$, where $S_{t}$ is the asset price on time t. $J_{t}^{y}$ is the jump, composed by the jump indicator $N_{t+1}^{y}$ and magnitude $\xi^{y} \sim N\left(\mu_{y}, \sigma_{y}^{2}\right)$. $\mu_{t}$ represents the equilibrium log-return of $y_{t}$ and, if $\sigma_{\mu}^{2}=0$, it is given by a constant, so that $\mu_{t}=\mu$ for all t.

$\gamma_{t}$ and $\delta_{t}$ are the mixture components for variance, in the same way proposed by the previous section. $\lambda_{t}^{-1}$ is the volatility of returns, and the main interest lays on estimating its value through time, since it is the main variable on risk and stock options pricing.

$\sigma_{\mu}^{2}$ is the variance of $\mu_{t} . \omega$ is a discount factor and is specified, in order to avoid needing to recur to $\mathrm{MH}$ algorithms for its estimation, keeping an automatic estimation procedure via Gibbs Sampling. $a_{t-1}$ is a form parameter of the filtering distribution for $\lambda_{t}$. 


\subsection{Bayesian Inference}

In this section it will be presented the priors and full conditional posteriors for each parameter. First it will be presented the parameters related to the DLM with mixtures on scale and then the parameters related to jumps. A detailed derivation of full conditional posterior distributions can be seen on Appendix A and Appendix B.

\subsubsection{NGSVJ related parameters: conditional posteriors}

For the log-returns mean parameter, $\mu_{t}=\theta_{t}$, a prior $\mathrm{N}\left(m_{0}, C_{0}\right)$ is specified and the samples can be obtained through a standard FFBS algorithm. In case it is defined as static, then $\sigma_{\mu}^{2}$ must be set zero, so that the error associated to $\mu_{t}$, $\omega_{t}$, will be a zero degenerate Normal, leading to $\mu_{t}=\mu$.

To simplify notation, let $\Phi=\left(\mu_{t}, J_{t}^{y}, \gamma_{t}, \delta_{t}, \lambda_{t}, \sigma_{u}^{2}\right)$, except variable in the index, i.e. $\Phi_{[-\lambda]}=\left(\mu_{t}, J_{t}^{y}, \gamma_{t}, \delta_{t}, \sigma_{u}^{2}\right)$.

For $\lambda_{t}$ a prior Gamma $\left(\omega a_{t-1}, \omega b_{t-1}\right)$ is defined. Following the method proposed by Gamerman et al. (2013) [10], the updating distribution is:

$$
p\left(\lambda_{t} \mid y_{t}, \Phi_{[-\lambda]}\right) \sim \operatorname{Gamma}\left(\omega a_{t-1}+\frac{1}{2}, \omega b_{t-1}+\gamma_{t} \frac{\left(Y_{t}-F_{t} \theta_{t}-J_{t}\right)^{2}}{2}\right)
$$

The $\mathrm{a}$ and $\mathrm{b}$ parameters are obtained through the filtering distribution in Gamerman et al. (2013) [10]. $\omega$ is a fixed discount factor. Appendix C shows the sampling algorithm used for sampling $\lambda_{1: n}$ using a smoothing procedure.

For $\sigma_{\mu}^{2}$ a InverseGamma $\left(a_{0}, b_{0}\right)$ non-informative prior is assumed. Appendix A3 show derivation details. The full conditional posterior distribution is:

$$
p\left(\sigma_{\mu}^{2} \mid y_{t}, \Phi_{\left[-\sigma_{\mu}^{2}\right]}\right) \sim \text { InverseGamma }\left(a_{0}+\frac{n}{2}, b_{0}+\frac{\sum_{i=2}^{t} \delta_{i}\left(\theta_{i}-\theta_{i-1}\right)^{2}}{2}\right)
$$


In case $\theta_{t}$ is defined as static, then $\sigma_{\mu}^{2}=0$.

For the mixture component $\gamma_{t}$ a prior $\operatorname{Gamma}\left(\frac{\nu}{2}, \frac{\nu}{2}\right)$ is defined, which, according to Gamerman et al. (2013) [10], when mixed as $\gamma_{t}^{-1}$, resulting in InverseGamma, leads to a Student-t with $\nu$ degrees of freedom to the innovations. Appendix A1 show derivation details. The full conditional posterior distribution is:

$$
p\left(\gamma_{t} \mid y_{t}, \Phi_{[-\gamma]}\right) \sim \operatorname{Gamma}\left(\frac{\nu}{2}+\frac{1}{2}, \frac{\nu}{2}+\lambda_{t} \frac{\left(Y_{t}-F_{t} \theta_{t}-J_{t}\right)^{2}}{2}\right)
$$

The same stands for the mixture $\delta_{t}$. Appendix A2 show derivation details. In this case, posterior is given by:

$$
p\left(\delta_{t} \mid y_{t}, \Phi_{[-\delta]}\right) \sim \operatorname{Gamma}\left(\frac{\nu}{2}+\frac{1}{2}, \frac{\nu}{2}+\frac{\left(\theta_{t}-\theta_{t-1}\right)\left(\sigma_{\mu}^{2}\right)^{-1}\left(\theta_{t}-\theta_{t-1}\right)}{2}\right)
$$

The parameter $\nu$ will not be estimated using MCMC methods, since it would lead to Metropolis based algorithms, as there is no closed form for its posterior. Instead, a sensibility analysis will be made, comparing the B-statistics, which will be defined on section 2.4.4 for different values of $\nu$. Recall that the main objective of the NGSV $i$ model is keeping an automatic and fast procedure for estimation, thus, such procedure will avoid the need to recur to computational intensive methods for estimating $\nu$.

\subsubsection{Jump related parameters}

Detailed derivation of the full conditionals shown in this topic can be seen on Appendix B. The jump sizes $\xi_{t+1}^{y}$ follow a $\mathrm{N}\left(\mu_{y}, \sigma_{y}^{2}\right)$. For the mean $\mu_{y}$ a noninformative prior $\mathrm{N}(m, v)$ is set, resulting in a full conditional posterior:

$$
p\left(\mu_{y} \mid y_{t}, \Phi_{\left[-\mu_{y}\right]}\right) \sim \mathrm{N}\left(\frac{m \sigma_{y}^{2}+v n_{j} \bar{\xi}^{y}}{\sigma_{y}^{2}+n_{j} v}, \frac{v \sigma_{y}^{2}}{\sigma_{y}^{2}+n_{j} v}\right)
$$

For the variance $\sigma_{y}^{2}$ a prior InverseGamma $(\alpha, \beta)$ is set, resulting in a full 
conditional posterior:

$$
p\left(\sigma_{y}^{2} \mid y_{t}, \Phi_{\left[-\sigma_{y}^{2}\right]}\right) \sim \text { InverseGamma }\left(\alpha+\frac{n_{j}}{2}, \beta+\frac{\sum_{\substack{i=1 \\ J_{i} \neq 0}}^{t}\left(\xi_{i+1}^{y}-\mu_{y}\right)^{2}}{2}\right)
$$

In both cases, $n_{j}$ is the number of times the jump is observed, and $\overline{\xi^{y}}$ the mean of its sizes $\xi^{y}$. As jump sizes are assumed to be Normal, the posterior is also Normal, with parameters:

$$
p\left(\xi_{t+1}^{y} \mid y_{t}, \Phi_{[-\xi]}\right) \sim N\left(\frac{\mu_{y} \gamma_{t}^{-1} \lambda_{t}^{-1}+y_{t} \sigma_{y}^{2}-F_{t} \theta_{t} \sigma_{y}^{2}}{\sigma_{y}^{2}+\gamma_{t}^{-1} \lambda_{t}^{-1}}, \frac{\sigma_{y}^{2} \gamma_{t}^{-1} \lambda_{t}^{-1}}{\sigma_{y}^{2}+\gamma_{t}^{-1} \lambda_{t}^{-1}}\right)
$$

For jump probabilities $\rho$, a prior $\operatorname{Beta}(\alpha, \beta)$ is set. The full conditional posterior is given by:

$$
p\left(\rho \mid y_{t}, \Phi_{[-\rho]}\right) \sim \operatorname{Beta}\left(\alpha+\sum_{i=0}^{n} N_{i}^{y}, \beta+n-\sum_{i=0}^{n} N_{i}^{y}\right)
$$

Since the jump indicator $N^{y}$ can assume only two values, 0 or 1 , the probability of observation $t+1$ be a jump is given by:

$$
P\left(N_{t+1}^{y}=1 \mid \Phi_{[-N]}\right) \propto \rho P\left(Y_{t+1} \mid N_{t+1}^{y}=1, \Phi_{[-N]}\right)
$$

which is easy to calculate, since the density $P\left(Y_{t+1} \mid N_{t+1}^{y}=1, \Phi_{[-N]}\right)$ can be calculated from a Normal distribution. Using the concept proposed by Brooks and Prokopczuk (2011) [3], if $P\left(N_{t+1}^{y}=1 \mid \Phi_{[-N]}\right)$ is greater then a threshold $\alpha$, then $N_{t+1}^{y}=1$. The threshold $\alpha$ is chosen such that the number of jumps identified corresponds to the estimate of the jump intensity $\rho$.

As said before, all conditional posterior distributions are known and have closed form, so it is possible to sample directly using a Gibbs Sampler instead of Metropolis steps. In order to preserve this structure, the degrees of freedom of the resulting Student-t distribution, $\nu$, is specified and a sensibility analysis is made in order to evaluate it, since posterior distribution for this parameter does not have closed form, and one of the objectives of using this model is avoiding computational intensive methods. For the same purpose, the discount factor, $\omega$, is also specified. This is particularly useful when dealing with high dimensional data. 
Since Metropolis uses an accept-reject algorithm, with several tunning parameters, it tends to be a much less efficient method for estimation than Gibbs Sampler. For practical applications on financial markets, the sooner information is available more time analysts will have to make their strategies and orient investors about capital allocation.

\subsubsection{MCMC Algorithm}

Let $Y_{n}=\left\{y_{t}\right\}_{t=1}^{n}, \theta=\left\{\theta_{t}\right\}_{t=1}^{n}, J=\left\{J_{t}^{y}\right\}_{t=1}^{n}=\left\{\xi_{t+1}^{y} N_{t+1}^{y}\right\}_{t=1}^{n}, \gamma=\left\{\gamma_{t}\right\}_{t=1}^{n}$, $\lambda=\left\{\lambda_{t}\right\}_{t=1}^{n}, \delta=\left\{\delta_{t}\right\}_{t=1}^{n}, \xi=\left\{\xi_{t+1}^{y}\right\}_{t=1}^{n}, N=\left\{N_{t+1}^{y}\right\}_{t=1}^{n}$ and prior probability density $\pi(\gamma), \pi\left(\sigma_{\mu}^{2}\right), \pi(\delta), \pi\left(\mu_{y}\right), \pi\left(\sigma_{y}^{2}\right), \pi(\xi), \pi\left(\rho_{y}\right)$ are set for $\gamma, \sigma_{\mu}^{2}, \delta, \mu_{y}, \sigma_{y}^{2}, \xi, \rho_{y}$. Then, a sample from the posterior distribution $\pi\left(\theta, \lambda, \gamma, \sigma_{\mu}^{2}, \delta, \mu_{y}, \sigma_{y}^{2}, J, \rho_{y} \mid Y_{n}\right)$ is drawn by the MCMC technique. The following sampling algorithm is followed:

1. Initialize $\theta^{(0)}, \lambda^{(0)}, \gamma^{(0)},\left(\sigma_{\mu}^{2}\right)^{(0)}, \delta^{(0)}, \mu_{y}^{(0)},\left(\sigma_{y}^{2}\right)^{(0)}, \xi^{(0)}, N^{(0)}$ and $\rho_{y}^{(0)}$.

2. Set $j=1$.

3. Sample $\theta^{(j)} \mid Y_{n}, J^{(j-1)}, \lambda^{(j-1)}, \gamma^{(j-1)}, \delta^{(j-1)},\left(\sigma_{\mu}^{2}\right)^{(j-1)}$ using FFBS algorithm.

4. Block sample $\lambda^{(j)} \mid Y_{n}, \theta^{(j)}, J^{(j-1)}, \gamma^{(j-1)}$ using algorithm described on Appendix C.

5. Block sample $\gamma^{(j)} \mid Y_{n}, \theta^{(j)}, J^{(j-1)}, \lambda^{(j)}$ as in Eq.(2.13).

6. Sample $\left(\sigma_{\mu}^{2}\right)^{(j)} \mid \theta^{(j)}, \delta^{(j-1)}$ as in Eq.(2.12).

7. Block sample $\delta^{(j)} \mid \theta^{(j)},\left(\sigma_{\mu}^{2}\right)^{(j)}$ as in Eq.(2.14).

8. Sample $\mu_{y}^{(j)} \mid \xi^{(j-1)},\left(\sigma_{y}^{2}\right)^{(j-1)}$ as in Eq.(2.15).

9. Sample $\left(\sigma_{y}^{2}\right)^{(j)} \mid \xi^{(j-1)}, \mu_{y}^{(j)}$ as in Eq.(2.16).

10. Block sample $J^{(j)} \mid Y_{n}, \theta^{(j)}, \lambda^{(j)}, \gamma^{(j)}, \mu_{y}^{(j)},\left(\sigma_{y}^{2}\right)^{(j)}$ by Block sample $\xi^{(j)} \mid Y_{n}, \theta^{(j)}, \lambda^{(j)}, \gamma^{(j)}, \mu_{y}^{(j)},\left(\sigma_{y}^{2}\right)^{(j)}$ as in Eq.(2.17). 
Block sample $N^{(j)} \mid Y_{n}, \theta^{(j)}, \lambda^{(j)}, \gamma^{(j)}, \xi^{(j)}$ as in Appendix B.5.

11. Sample $\rho_{y}^{(j)} \mid J^{(j)}$ as in Eq.(2.18).

12. Set $j=j+1$.

13. If $j \leq M$, go to 3 , otherwise stop.

Since all conditional posterior distributions have closed form, the technique used is the Gibbs Sampler. Appendix A, Appendix B and Appendix C brings more details on conditional posterior distributions used for sampling and the method for sampling $\lambda$.

\subsubsection{Model Diagnostics and Specification Tests}

The approach to compare different specifications for model parameters is the B-statistic criteria. According to Ibrahim et al. (2001) [12], cited by Demarqui (2010) [5], this approach uses the average of the logarithm of the pseudo-marginal likelihood as a measure to assess the goodness of fit of the models to be compared. This measure is based on the conditional predictive ordinate (CPO) statistic, and is defined as:

$$
B=\frac{1}{n} \sum_{i=1}^{n} \log \left(C P O_{i}\right)
$$

where $\mathrm{n}$ is the number of observations and $C P O_{i}$ corresponds to the posterior predictive density of $y_{i}$. It can be well approximated by:

$$
C \hat{P} O_{i}=M\left\{\sum_{l=1}^{M}\left[L\left(y_{i} \mid \mu^{l}, J^{l}, \gamma^{l}, \lambda^{l}\right)\right]^{-1}\right\}^{-1}
$$

where $\mathrm{L}$ is the likelihood function, $\left(\mu^{l}, J^{l}, \gamma^{l}, \lambda^{l}\right)$ corresponds to the l-th draw of the posterior distribution $\pi\left(\mu^{l}, J^{l}, \gamma^{l}, \lambda^{l} \mid D\right), l=1, \ldots, M$, and $M$ the size of the posterior sampled distribution of the parameters.

It is desirable to obtain the highest B-statistic possible with the parameters specification. 


\section{Chapter 3}

\section{Simulations}

This chapter will discuss the model results for simulated data. The first simulation set was made using Gaussian distribution and its main goal is to check the model capability of estimating the jump parameters, as well as understand how the choice of the model hyperparameters, threshold $\alpha$ and degrees of freedom $\nu$, influences the estimates.

The second simulation set was made using a Student's t distribution and the main goal is to check how heavier tails influence the model capability of estimating jump parameters and how a degree of freedom, $\nu$, choice affects the model estimation.

\subsection{Procedure}

For all Gaussian simulation scenarios, one series with 1,000 observations was generated from:

$$
y_{t}=\mu+J_{t}^{y}+v_{t}, \quad v_{t} \sim N\left(0, \lambda^{-1}\right)
$$

where $\mu$ and $\lambda^{-1}$ were considered static parameters, and their values were the 
same for all scenarios. Table 3.2 shows a summary of their values and simulated scenarios.

Jump components $\xi^{y}$ and $N^{y}$ were generated from a $N\left(\mu_{y}, \sigma_{y}^{2}\right)$ and Bernoulli $\left(\rho_{y}\right)$ respectively. In each scenario, different values for $\mu_{y}$ and $\sigma_{y}^{2}$ were chosen, but $\rho_{y}$ was the same. In the scenario of manually imputed jumps, together with this structure, jumps were manually imputed according to Table 3.1.

\begin{tabular}{|l||l|l|}
\hline Position (t) & Jump size $\xi^{y}$ & $N^{y}$ \\
\hline 100 & 15 & 1 \\
200 & -20 & 1 \\
400 & -8 & 1 \\
500 & -30 & 1 \\
600 & 25 & 1 \\
800 & -10 & 1 \\
900 & -40 & 1 \\
\hline
\end{tabular}

Table 3.1: Manually imputed jumps

For Student's-t scenarios, 1,000 observations were generated from:

$$
y_{t}=\mu+J_{t}^{y}+\epsilon_{t}, \quad \epsilon_{t} \sim t_{20}\left(0, \lambda^{-1}\right)
$$

where $\mu$ and $\lambda^{-1}$ were considered static parameters, and their value was the same used in Gaussian scenarios for jump components. 


\begin{tabular}{|l|l|l|l|l|l|l|}
\hline \multicolumn{2}{|l|}{ Gaussian Scenarios } & \multicolumn{4}{l|}{ Student's t Scenarios } \\
\hline \begin{tabular}{l}
$\mid \begin{array}{l}y_{t}=\mu+J_{t}^{y}+v_{t}, \\
v_{t} \sim N\left(0, \lambda^{-1}\right)\end{array}$ \\
\hline
\end{tabular}$y_{t}=\mu+J_{t}^{y}+\epsilon_{t}$, \\
\hline $\begin{array}{l}\text { Scenario } \\
\epsilon_{t} \sim t_{20}\left(0, \lambda^{-1}\right)\end{array}$ \\
\hline Strong Jumps & 0.10 & - & 0.5 & 0.05 & -3.0 & 4 \\
\hline Weak Jumps & 0.10 & - & 0.5 & 0.05 & -1.5 & 1 \\
\hline $\begin{array}{l}\text { Man. Imp. } \\
\text { Jumps }\end{array}$ & 0.10 & - & 0.5 & 0.05 & -3.69 & 8.67 \\
\hline $\begin{array}{l}\text { Vol. Chang- } \\
\text { ing }\end{array}$ & 0.10 & - & $0.5 \leftrightarrow 5$ & 0.05 & -3.0 & 4 \\
\hline
\end{tabular}

Table 3.2: Summary of Simulation Scenarios

\subsection{Results and comments}

Simulation results for each scenario are discussed in this section.

For model hyperparameters, the discount factor $\omega$ was fixed as $0.9, \alpha$ and $\nu$ vary according do simulation scenario. A $\mathrm{G}\left(\frac{\nu}{2}, \frac{\nu}{2}\right)$ prior distribution was specified for $\gamma_{t}$ in order to obtain the Student's $t_{\nu}$-errors for the observation and system disturbances. In order to keep the model comparable to Eraker et al. (2003) [9], $\theta=\mu$ component was set to be fixed over time, so that $\sigma_{\mu}^{2}=0$. For the mean components, $\mu$ and $\mu_{y}, \mathrm{~N}(0,100)$ priors were specified and for $\sigma_{y}^{2}$ a InverseGamma(0.1,0.1) prior. Also, $a_{0}=0.1$ and $b_{0}=0.1$, as suggested in West et al. (1987,p. 333), cited by Gamerman et al. (2013) [10]. A Beta $(2,40)$ prior distribution was specified to $\rho_{y}$, as in Eraker et al. (2003) [9].

The results were obtained with a 100,000 iteration chain, burning 60,000 observations, with a lag of 30 observations, resulting in 1,291 observations. MCMC chains convergence was verified through informal graphic methods. All programing was done in the Ox software (Doornik, 2008) [6]. The $\mathrm{R}$ software (The $\mathrm{R}$ foundation for Statistical Computing, 2015) [23] was used for plotting graphics. 
Machine used was an Intel Core i5 - $2310 \mathrm{CPU}$ at $2.90 \mathrm{Ghz}, 4$ GB RAM, and 64 bit Windows Seven operating system.

\subsubsection{Simulations for the Gaussian approach}

Three simulation scenarios were made using Normal distribution. The first has strong jumps, the second has weak jumps and the third includes manually imputed jumps.

Tables 3.3, 3.4 and 3.5 show true and estimated values for each scenario. Point estimates are the posterior mean and, inside brackets, are the standard deviation of posterior distribution. For each of three scenarios, the model parameter $\nu$ was set as 100,20 or 5 , so that smaller values give more flexibility to the model to capture heavy tails. The threshold $\alpha$ was set as $0.70,0.80$ or 0.90 , and gives the probability cut-point to an observation to be considered a jump.

For data generated from a Normal distribution, results suggests that the best option is to give low flexibility to the model through $\nu$ parameter. As $\nu$ decreases, $\gamma^{-1}$ component distances itself from 1 , value that would indicate that the model assumes a normal distribution for innovations. Giving flexibility to the model through $\nu$ can also influence the jump components estimates, since some jumps can be identified by the model as a mere heavy tail, thus reducing the number of jumps identified by the model, resulting in lower jump probability, $\rho_{y}$, and higher jump means and standard deviation, $\mu_{y}$ and $\sigma_{y}$, since they are estimated based on observations that were considered jumps by the model, so that only the bigger outliers will be considered jumps. 


\begin{tabular}{|c|c|c|c|c|}
\hline \multicolumn{5}{|c|}{$\nu=100$} \\
\hline Parameters & True & $\alpha=0.70$ & $\alpha=0.80$ & $\alpha=0.90$ \\
\hline$\mu$ & 0.10 & $0.1085(0.0245)$ & $0.1054(0.0245)$ & $0.1013(0.0245)$ \\
\hline$\lambda^{-1}$ & 0.5 & $0.5196(0.1449)$ & $0.5441(0.1526)$ & $0.5687(0.1664)$ \\
\hline$\gamma^{-1}$ & & $0.9997(0.0124)$ & $1.0001(0.0069)$ & $1.0003(0.0136)$ \\
\hline$\rho_{y}$ & 0.05 & $0.0406(0.0061)$ & $0.0346(0.0057)$ & $0.0283(0.0052)$ \\
\hline$\mu_{y}$ & -3 & $-2.8917(0.8207)$ & $-3.3448(0.8737)$ & $-3.8142(1.0526)$ \\
\hline$\sigma_{y}$ & 4 & $4.8558(5.5912)$ & $5.0687(7.1876)$ & $5.4448(9.2952)$ \\
\hline B & & -2.1860 & -2.1316 & -2.1590 \\
\hline \multicolumn{5}{|c|}{$\nu=20$} \\
\hline Parameters & True & $\alpha=0.70$ & $\alpha=0.80$ & $\alpha=0.90$ \\
\hline$\mu$ & 0.10 & $0.1026(0.0245)$ & $0.1007(0.0100)$ & $0.0949(0.0100)$ \\
\hline$\lambda^{-1}$ & 0.5 & $0.516(0.1442)$ & $0.5335(0.01546)$ & $0.5624(0.1744)$ \\
\hline$\gamma^{-1}$ & & $0.9999(0.0558)$ & $1.0002(0.0577)$ & $1.0000(0.0599)$ \\
\hline$\rho_{y}$ & 0.05 & $0.0360(0.0058)$ & $0.0307(0.0054)$ & $0.0257(0.0050)$ \\
\hline$\mu_{y}$ & -3 & $-3.1681(0.8985)$ & $-3.5272(1.0151)$ & $-3.9760(1.1091)$ \\
\hline$\sigma_{y}$ & 4 & $5.0432(7.6764)$ & $5.3023(8.6246)$ & $5.5548(10.2356)$ \\
\hline $\mathrm{B}$ & & -2.8880 & -2.8168 & -3.4564 \\
\hline \multicolumn{5}{|c|}{$\nu=5$} \\
\hline Parameters & True & $\alpha=0.70$ & $\alpha=0.80$ & $\alpha=0.90$ \\
\hline$\mu$ & 0.10 & $0.0343(0.0574)$ & $0.0270(0.0557)$ & $0.0248(0.0539)$ \\
\hline$\lambda^{-1}$ & 0.5 & $0.7039(0.3530)$ & $0.7141(0.3619)$ & $0.7224(0.3707)$ \\
\hline$\gamma^{-1}$ & & $1.0173(0.1860)$ & $1.0181(0.1890)$ & $1.0175(0.1954)$ \\
\hline$\rho_{y}$ & 0.05 & $0.0166(0.0041)$ & $0.0126(0.0036)$ & $0.0090(0.0032)$ \\
\hline$\mu_{y}$ & -3 & $-3.2192(1.6955)$ & $-3.7722(2.0005)$ & $-4.635(2.5715)$ \\
\hline$\sigma_{y}$ & 4 & $5.0499(14.7474)$ & $5.2446(23.4603)$ & $5.5002(36.8306)$ \\
\hline $\mathrm{B}$ & & -4.7795 & -4.6739 & -4.07547 \\
\hline
\end{tabular}

Table 3.3: Simulation 1 - Normal with Strong Jumps 


\begin{tabular}{|c|c|c|c|c|}
\hline \multicolumn{5}{|c|}{$\nu=100$} \\
\hline Parameters & True & $\alpha=0.70$ & $\alpha=0.80$ & $\alpha=0.90$ \\
\hline$\mu$ & 0.10 & $0.0838(0.0245)$ & $0.0816(0.0245)$ & $0.0777(0.0245)$ \\
\hline$\lambda^{-1}$ & 0.5 & $0.5463(0.1552)$ & $0.5765(0.1513)$ & $0.6001(0.0257)$ \\
\hline$\gamma^{-1}$ & & $0.9997(0.0128)$ & $0.9998(0.0134)$ & $0.9997(0.0139)$ \\
\hline$\rho_{y}$ & 0.05 & $0.0224(0.0047)$ & $0.0135(0.0037)$ & $0.0086(0.0031)$ \\
\hline$\mu_{y}$ & -1.5 & $-1.7584(0.5561)$ & $-2.6896(0.6550)$ & $-3.2649(0.5548)$ \\
\hline$\sigma_{y}$ & 1 & $2.1459(1.9775)$ & $1.5889(2.4945)$ & $1.1317(1.8626)$ \\
\hline $\mathrm{B}$ & & -1.7125 & -1.6837 & -1.6319 \\
\hline \multicolumn{5}{|c|}{$\nu=20$} \\
\hline Parameters & True & $\alpha=0.70$ & $\alpha=0.80$ & $\alpha=0.90$ \\
\hline$\mu$ & 0.10 & $0.0796(0.0265)$ & $0.0766(0.0245)$ & $0.0726(0.0265)$ \\
\hline$\lambda^{-1}$ & 0.5 & $0.5383(0.1411)$ & $0.5567(0.1442)$ & $0.5736(0.1520)$ \\
\hline$\gamma^{-1}$ & & $0.9999(0.0578)$ & $0.9998(0.0600)$ & $0.9994(0.0622)$ \\
\hline$\rho_{y}$ & 0.05 & $0.017(0.0041)$ & $0.0109(0.0034)$ & $0.0068(0.0028)$ \\
\hline$\mu_{y}$ & -1.5 & $-2.0978(0.6472)$ & $-2.8237(0.6353)$ & $-3.2626(0.0624)$ \\
\hline$\sigma_{y}$ & 1 & $1.9467(2.4967)$ & $1.5501(3.7867)$ & $36.1164\left(3.87 \times 10^{4}\right)$ \\
\hline $\mathrm{B}$ & & -2.1188 & -2.0660 & -1.9050 \\
\hline \multicolumn{5}{|c|}{$\nu=5$} \\
\hline Parameters & True & $\alpha=0.70$ & $\alpha=0.80$ & $\alpha=0.90$ \\
\hline$\mu$ & 0.10 & $0.0650(0.0316)$ & $0.0672(0.0300)$ & $0.0743(0.0283)$ \\
\hline$\lambda^{-1}$ & 0.5 & $0.4816(0.1196)$ & $0.4813(0.1192)$ & $0.4765(0.1170)$ \\
\hline$\gamma^{-1}$ & & $1.0061(0.1895)$ & $1.0051(0.1944)$ & $1.0028(0.1998)$ \\
\hline$\rho_{y}$ & 0.05 & $0.0103(0.0033)$ & $0.0065(0.0028)$ & $0.0031(0.0022)$ \\
\hline$\mu_{y}$ & -1.5 & $-1.5721(1.2964)$ & $-1.7153(2.2590)$ & $-1.4104(4.9927)$ \\
\hline$\sigma_{y}$ & 1 & $2.3876(10.6410)$ & $4.30 \times 10^{7}$ & $4.01 \times 10^{11}$ \\
\hline & & & $\left(5.59 \times 10^{16}\right)$ & $\left(4.85 \times 10^{24}\right)$ \\
\hline $\mathrm{B}$ & & -3.8856 & -3.6652 & -2.9516 \\
\hline
\end{tabular}

Table 3.4: Simulation 2 - Normal with Weak Jumps 


\begin{tabular}{|c|c|c|c|c|}
\hline \multicolumn{5}{|c|}{$\nu=100$} \\
\hline Parameters & True & $\alpha=0.70$ & $\alpha=0.80$ & $\alpha=0.90$ \\
\hline$\mu$ & 0.10 & $0.1026(0.0245)$ & $0.0999(0.0245)$ & $0.0949(0.0245)$ \\
\hline$\lambda^{-1}$ & 0.5 & $0.5157(0.1473)$ & $0.5341(0.1483)$ & $0.5584(0.1565)$ \\
\hline$\gamma^{-1}$ & & $0.9997(0.0124)$ & $0.9997(0.0130)$ & $0.9999(0.0133)$ \\
\hline$\rho_{y}$ & 0.05 & $0.0455(0.0065)$ & $0.0402(0.0061)$ & $0.0352(0.0057)$ \\
\hline$\mu_{y}$ & -3.69 & $-4.1425(1.3731)$ & $-4.6041(1.5686)$ & $-4.9769(1.7611)$ \\
\hline$\sigma_{y}$ & 8.67 & $9.4938(20.6795)$ & $9.9238(23.8756)$ & $10.5485(29.2459)$ \\
\hline B & & -0.2039 & -0.2191 & -0.2179 \\
\hline \multicolumn{5}{|c|}{$\nu=20$} \\
\hline Parameters & True & $\alpha=0.70$ & $\alpha=0.80$ & $\alpha=0.90$ \\
\hline$\mu$ & 0.10 & $0.0964(0.0245)$ & $0.0859(0.0283)$ & $0.0703(0.0300)$ \\
\hline$\lambda^{-1}$ & 0.5 & $0.5121(0.1418)$ & $0.5700(0.2488)$ & $0.6093(0.2364)$ \\
\hline$\gamma^{-1}$ & & $0.9992(0.0549)$ & $0.9999(0.0573)$ & $0.9996(0.0614)$ \\
\hline$\rho_{y}$ & 0.05 & $0.0419(0.0062)$ & $0.0349(0.0057)$ & $0.0278(0.0052)$ \\
\hline$\mu_{y}$ & -3.69 & $-4.261(1.5869)$ & $-4.801(1.7731)$ & $-5.3818(2.3785)$ \\
\hline$\sigma_{y}$ & 8.67 & $9.8403(23.1824)$ & $10.5741(33.1793)$ & $11.9052(49.3205)$ \\
\hline $\mathrm{B}$ & & -0.2953 & -0.3921 & -0.3369 \\
\hline \multicolumn{5}{|c|}{$\nu=5$} \\
\hline Parameters & True & $\alpha=0.70$ & $\alpha=0.80$ & $\alpha=0.90$ \\
\hline$\mu$ & 0.10 & $0.0047(0.0624)$ & $0.0079(0.0624)$ & $0.0123(0.0592)$ \\
\hline$\lambda^{-1}$ & 0.5 & $0.7969(0.5517)$ & $0.8016(0.5534)$ & $0.7877(0.5302)$ \\
\hline$\gamma^{-1}$ & & $1.0176(0.1917)$ & $1.0165(0.1957)$ & $1.0160(0.2017)$ \\
\hline$\rho_{y}$ & 0.05 & $0.0162(0.0041)$ & $0.0122(0.0036)$ & $0.0088(0.0031)$ \\
\hline$\mu_{y}$ & -3.69 & $-3.5916(2.0887)$ & $-3.8937(3.0522)$ & $-4.5051(3.5466)$ \\
\hline$\sigma_{y}$ & 8.67 & $6.4283(46.7027)$ & $7.9884(77.5201)$ & $29.3070\left(2.19 \times 10^{4}\right)$ \\
\hline $\mathrm{B}$ & & -1.0225 & -1.0512 & -0.9017 \\
\hline
\end{tabular}

Table 3.5: Simulation 3 - Normal with Manually Imputed Jumps 


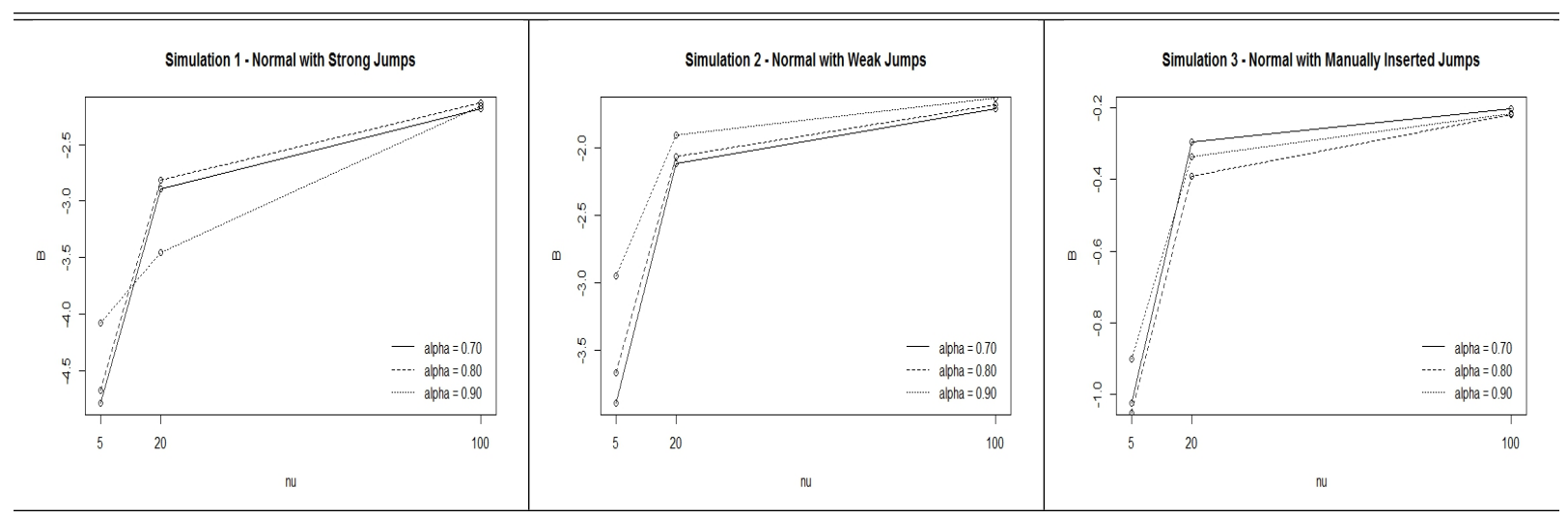

Figure 3.1: Normal Simulations - B-statistic comparison

Figure 3.1 shows a comparison between B-statistics for each simulation scenario. In all cases a higher $\nu$ gave a better model fit. On the other hand, for each case a different choice threshold $\alpha$ gave a better fit. Overall $\alpha=0.7$ seems to be a good choice, since its results are reasonably good for both strong and manually imputed jumps, being the worst choice only for weak jumps, where the model is known to have difficult on estimating parameters independently of parameters choice.

Figures 3.2, 3.3 and 3.4 show jumps probabilities and sizes for each simulation scenario, given $\nu=100$. They allow to see graphically the influence of threshold $\alpha$ on estimating jump probabilities and sizes. The light gray line is the true value and the superimposed black line is the value estimated by the model. 


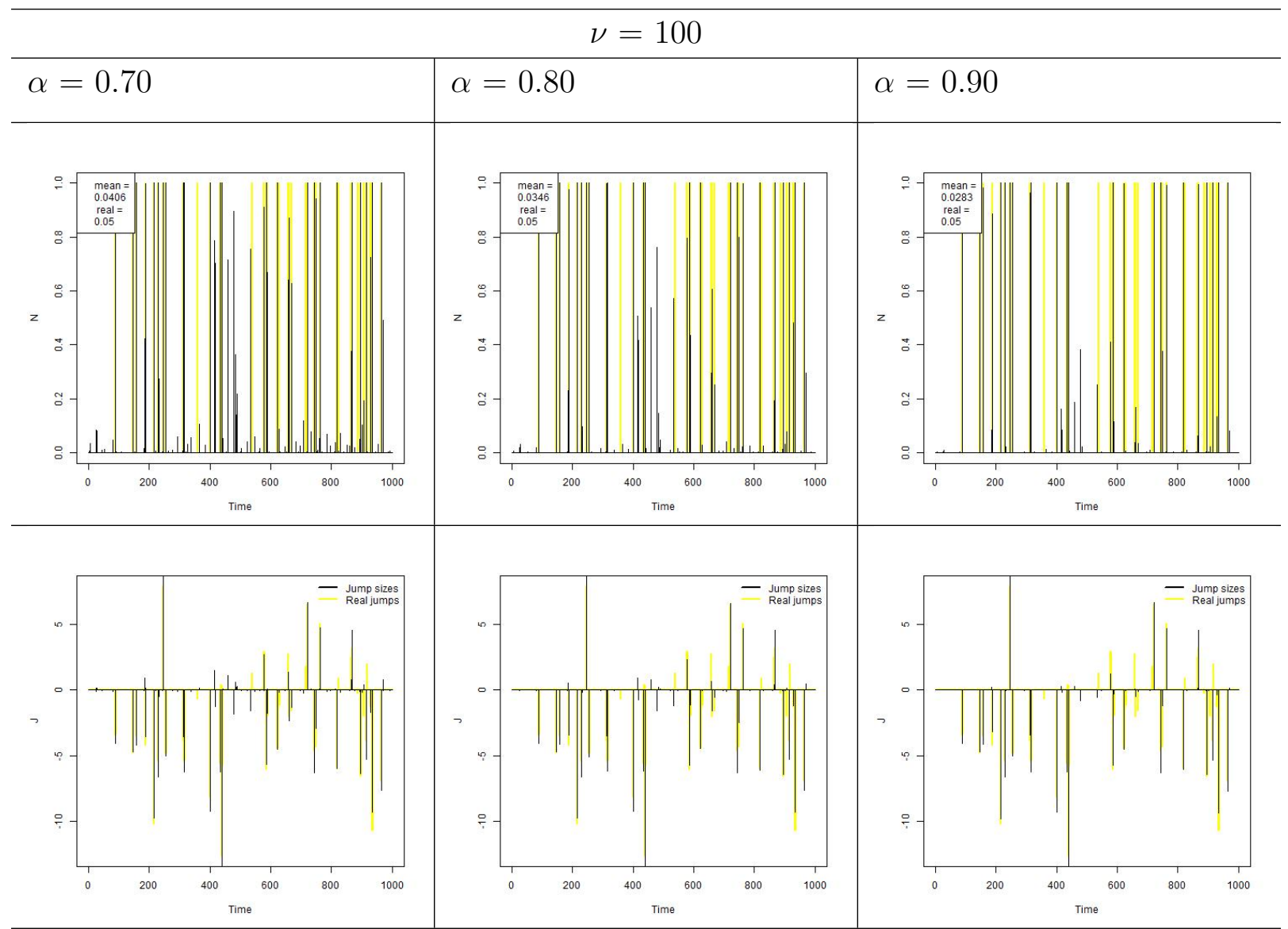

Figure 3.2: Simulation 1 - Normal with Strong Jumps - Jump probabilities and sizes

For strong jumps, Figure 3.2, it is possible to see that as the threshold $\alpha$ increases less observations are considered jumps, thus reducing estimate for jump probability mean. Higher $\alpha$ values tend to capture only the stronger outliers, leaving smaller jumps behind. 


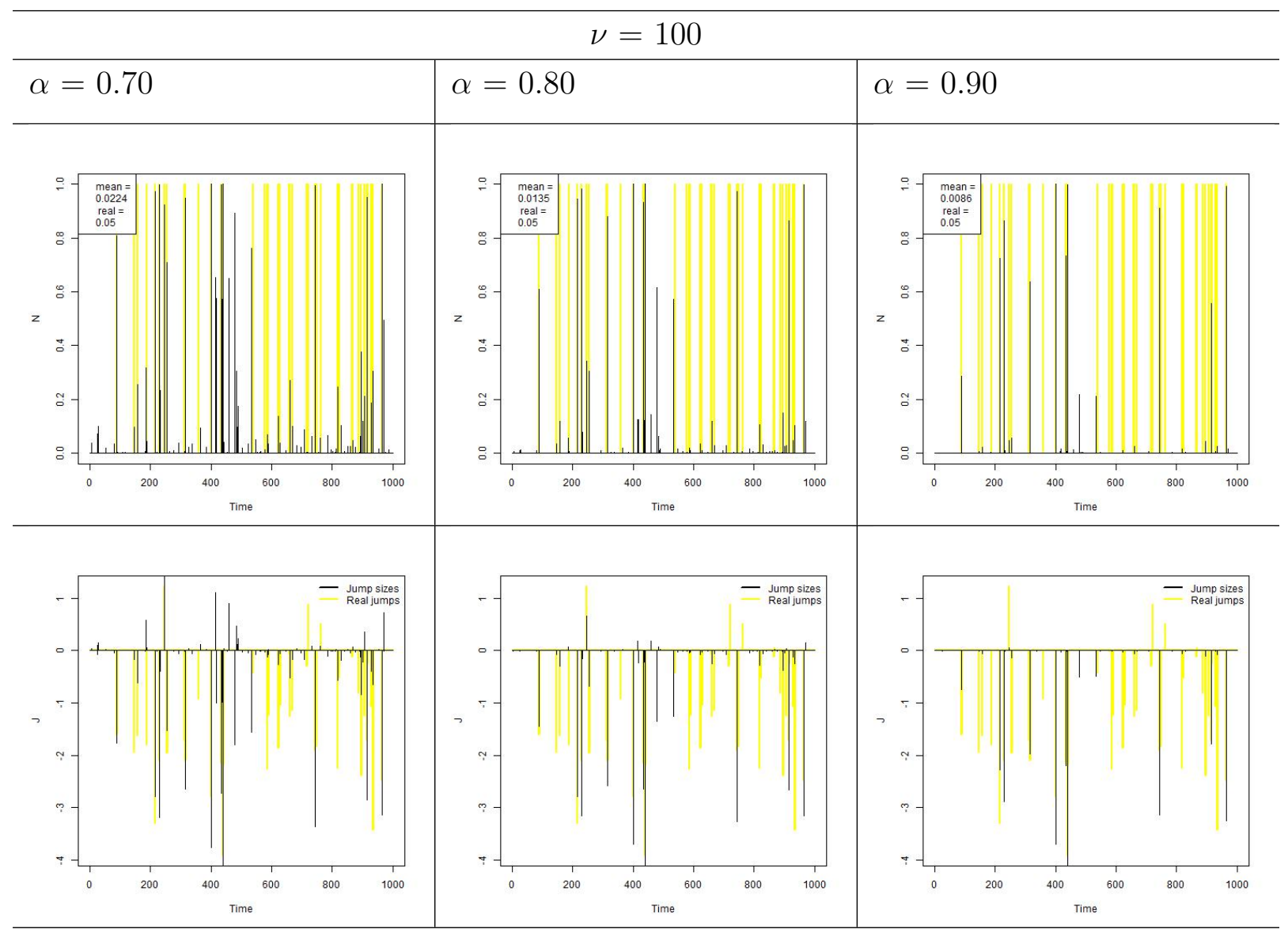

Figure 3.3: Simulation 2 - Normal with Weak Jumps - Jump probabilities and sizes

For weak jumps, Figure 3.3, it is possible to see that, independently of the choice of $\alpha$, the model has difficult on estimating jump probabilities and sizes. Only the biggest jumps are considered by the model, which explains the higher $\mu_{y}$ and $\sigma_{y}$ estimates seen on Table 3.4. Since the model uses the observations that were actually considered jumps to estimate these parameters, the sample obtained by the model for estimation is not representative for jumps as a whole, since only the stronger ones are taken in account. This leads to a jump mean estimative that is higher than the real one and also a higher standard deviation. 


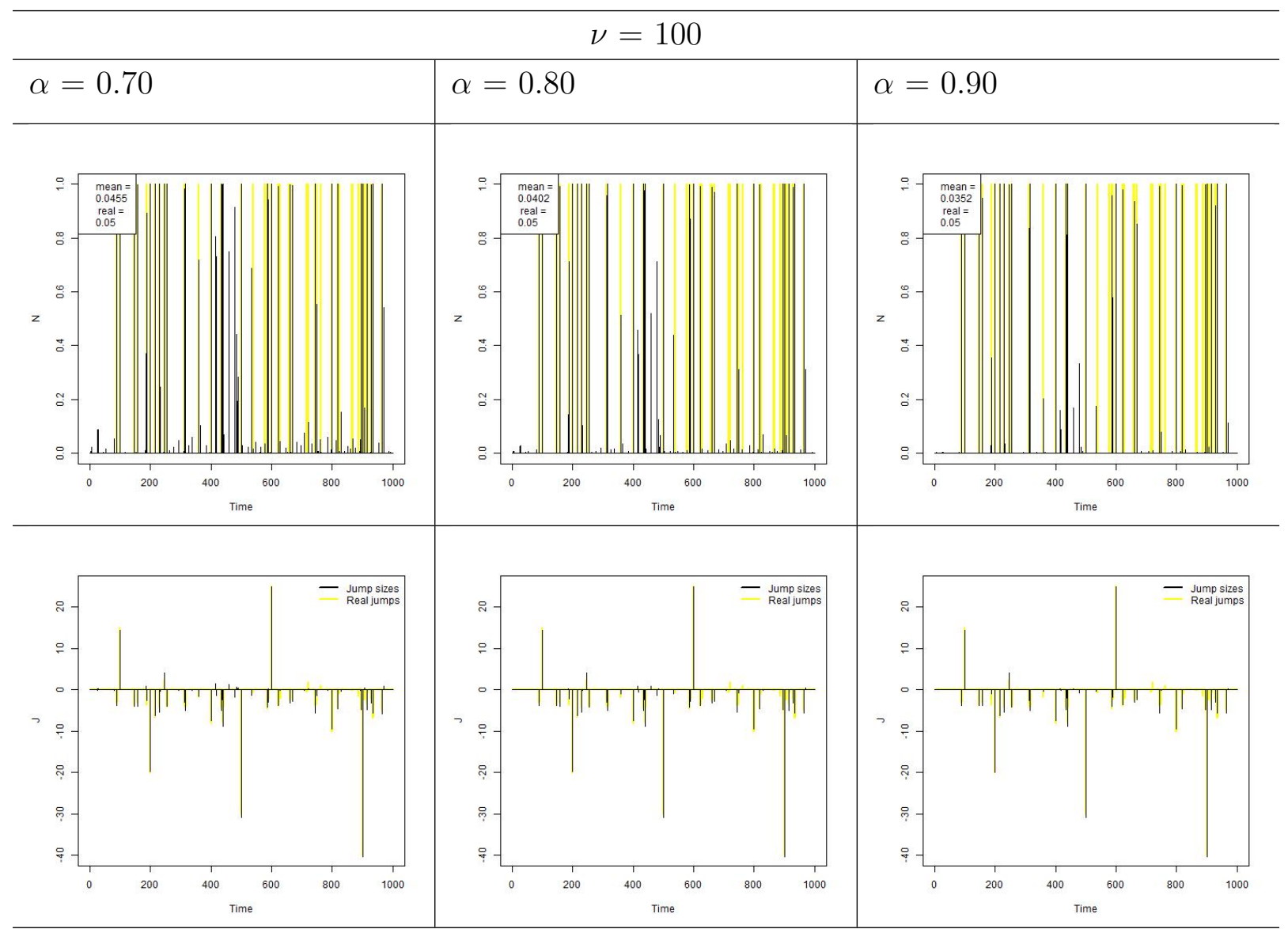

Figure 3.4: Simulation 3 - Normal with Manually Imputed Jumps - Jump probabilities and sizes

For manually imputed jumps, Figure 3.4, results were very similar to strong jumps, again giving the lead to $\alpha=0.70$, since gives jump probability estimate closer to the true. The model was able to capture all the manually imputed jumps, no matter what was the choice of $\alpha$.

\subsubsection{Sudent's t Simulations}

Three simulations scenarios were made using Student's t distribution with 20 degrees of freedom. First has strong jumps, second has weak jumps and third 
includes manually imputed jumps.

A forth simulation was made in order to verify how volatility changes affects jump related parameters estimation. For this case, model parameters were fixed at $\nu=30$ and $\alpha=0.7$, as suggests the previous simulation results. In this simulation, three scenarios were built: first on which true $\lambda^{-1}$ assumes 0.5 ; second with true $\lambda^{-1}=5$; third were true $\lambda^{-1}$ assumes 0.5 for certain periods of time and 5 for others. 


\begin{tabular}{|c|c|c|c|c|}
\hline \multicolumn{5}{|c|}{ Strong Jumps } \\
\hline Parameters & True & $\nu=10$ & $\nu=20$ & $\nu=30$ \\
\hline$\mu$ & 0.10 & $0.0592(0.0346)$ & $0.0776(0.0245)$ & $0.0806(0.0224)$ \\
\hline$\lambda^{-1}$ & 0.5 & $0.5910(0.2243)$ & $0.5383(0.1503)$ & $0.5353(0.1500)$ \\
\hline$\gamma^{-1}$ & & $1.0031(0.1068)$ & $1.0001(0.0557)$ & $0.9995(0.0387)$ \\
\hline$\rho_{y}$ & 0.05 & $0.0297(0.0053)$ & $0.0382(0.0060)$ & $0.0405(0.0061)$ \\
\hline$\mu_{y}$ & -3 & $-2.8919(1.0753)$ & $-2.8134(0.8941)$ & $-2.7093(0.8355)$ \\
\hline$\sigma_{y}$ & 4 & $5.1603(10.1587)$ & $4.8859(6.0511)$ & $4.8445(6.0036)$ \\
\hline B & & -2.9944 & -3.0956 & -2.3654 \\
\hline \multicolumn{5}{|c|}{ Weak Jumps } \\
\hline Parameters & True & $\nu=10$ & $\nu=20$ & $\nu=30$ \\
\hline$\mu$ & 0.10 & $0.0491(0.0300)$ & $0.0603(0.0245)$ & $0.0681(0.0245)$ \\
\hline$\lambda^{-1}$ & 0.5 & $0.5385(0.1371)$ & $0.5534(0.1466)$ & $0.5592(0.1507)$ \\
\hline$\gamma^{-1}$ & & $1.0019(0.1091)$ & $1.0000(0.0592)$ & $0.9998(0.0412)$ \\
\hline$\rho_{y}$ & 0.05 & $0.0138(0.0038)$ & $0.0177(0.0042)$ & $0.0191(0.0044)$ \\
\hline$\mu_{y}$ & -1.5 & $-2.1209(0.6937)$ & $-2.3737(0.4834)$ & $-2.4673(0.3964)$ \\
\hline$\sigma_{y}$ & 1 & $3.176(4.2308)$ & $1.7956(1.7863)$ & $1.1639(1.2341)$ \\
\hline B & & -2.6069 & -2.0139 & -1.8102 \\
\hline \multicolumn{5}{|c|}{ Manually Imputed Jumps } \\
\hline Parameters & True & $\nu=10$ & $\nu=20$ & $\nu=30$ \\
\hline$\mu$ & 0.10 & $0.0354(0.0387)$ & $0.0674(0.0265)$ & $0.0716(0.0245)$ \\
\hline$\lambda^{-1}$ & 0.5 & $0.7087(0.7638)$ & $0.5559(0.2057)$ & $0.5340(0.1487)$ \\
\hline$\gamma^{-1}$ & & $1.0026(0.1082)$ & $0.9999(0.0574)$ & $0.9994(0.0387)$ \\
\hline$\rho_{y}$ & 0.05 & $0.0299(0.0053)$ & $0.0404(0.0061)$ & $0.0429(0.0063)$ \\
\hline$\mu_{y}$ & -3.69 & $-3.7001(2.0452)$ & $-4.0406(1.5861)$ & $-3.9569(1.5381)$ \\
\hline$\sigma_{y}$ & 8.67 & $10.1371(38.7786)$ & $9.7148(25.3517)$ & $9.5976(22.8319)$ \\
\hline B & & -0.5748 & -0.3388 & -0.2388 \\
\hline
\end{tabular}

Table 3.6: Simulation 4 - Student's t - 20 degrees of freedom

For all simulations threshold $\alpha$ was fixes as 0.7 . Table 3.6 shows the results for simulations. Even though $\nu=20$ corresponds to a Student's t distribution with 20 degrees of freedom, $\nu=30$ shows point estimates closer to true values and a higher B-statistic for all cases. Because giving more flexibility to the model 
disrupts the capability of the model to identify jumps, it seems that a slightly higher value for $\nu$ can improve model sensibility for jumps.

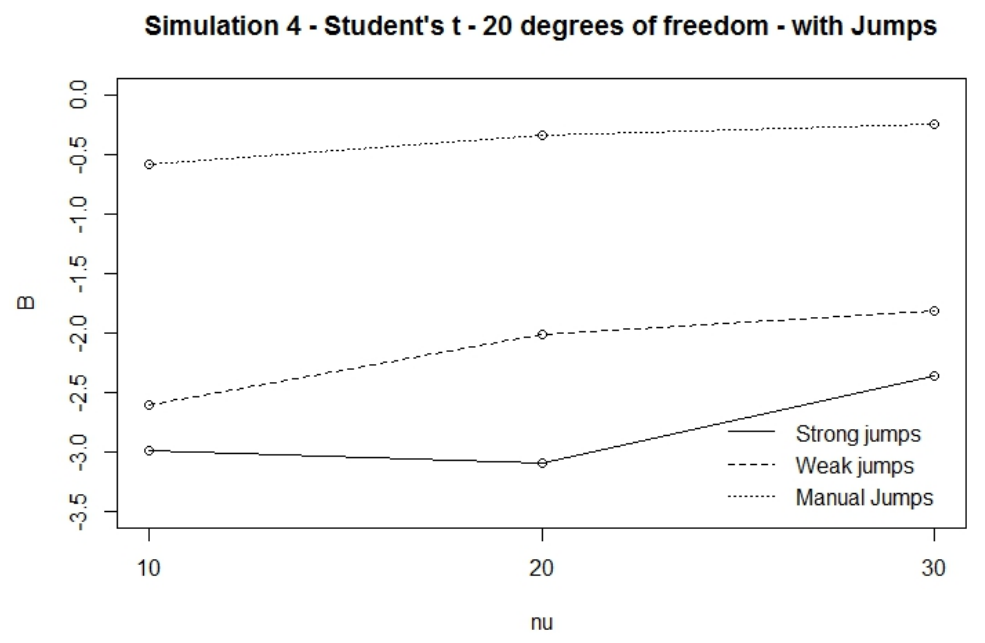

Figure 3.5: B-statistics comparison for Student's t scenario

Figure 3.5 shows the comparison between B-statistics for each scenario. For all cases $\nu=30$ has a higher B-statistic. Curiously, for strong jumps scenario, $\nu=10$ had a higher B-statistic than $\nu=20$. This suggests that, on giving more flexibility to the model, some jumps were considered by the model as part of the distribution tail, giving a better fit than considering them as jumps itself, since, as can be seen on Figure 3.6, the model underestimated most of jump sizes when using $\nu=10$. 


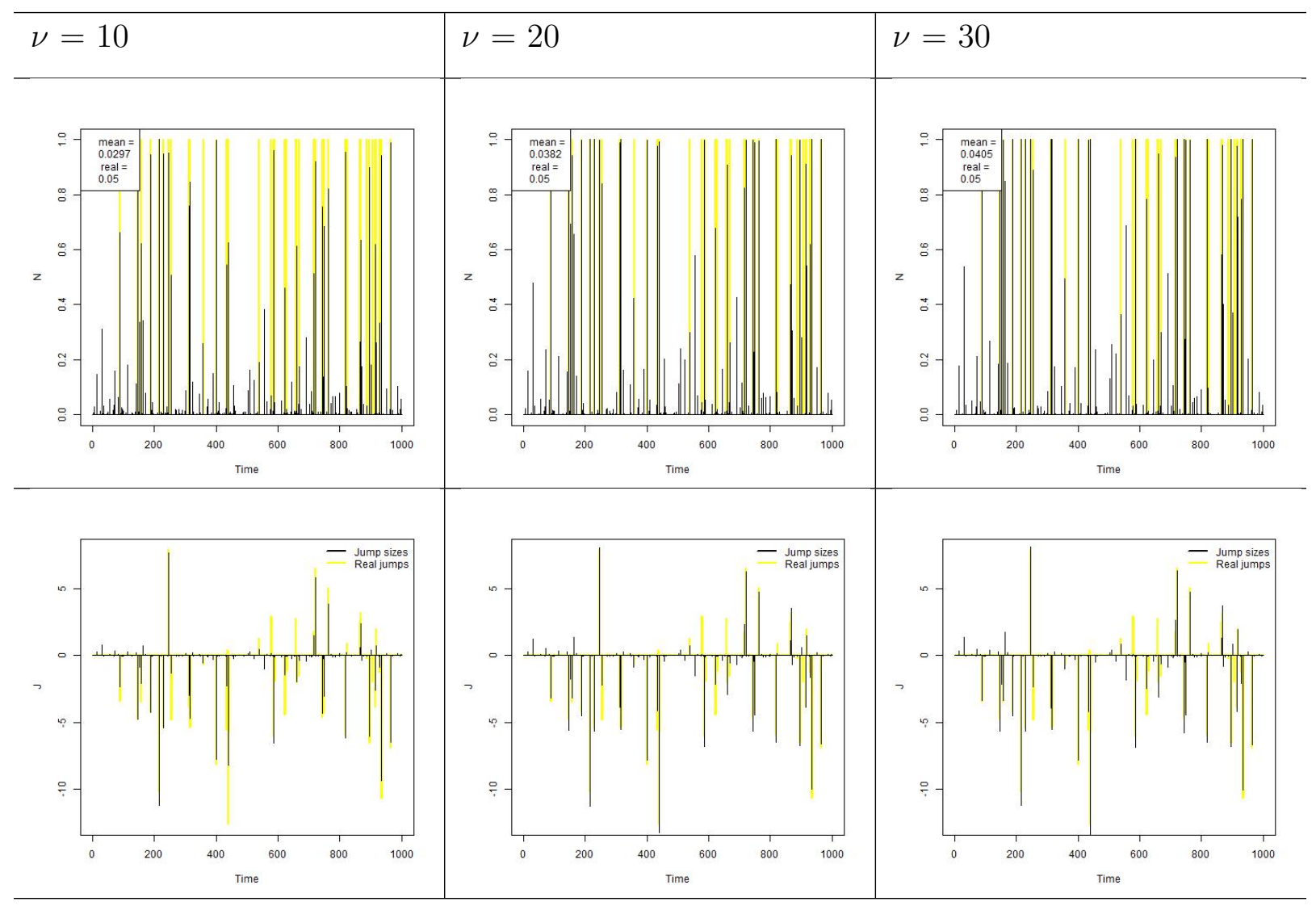

Figure 3.6: Simulation 4 - Strong Jumps - Jump probabilities and sizes

For strong jumps, Figure 3.6, results are similar to the observed on Gaussian simulations. Using a higher value for $\nu$ gives the model a better perception for jumps, since smaller values tend to classify smaller jumps as part of the distribution tail. 


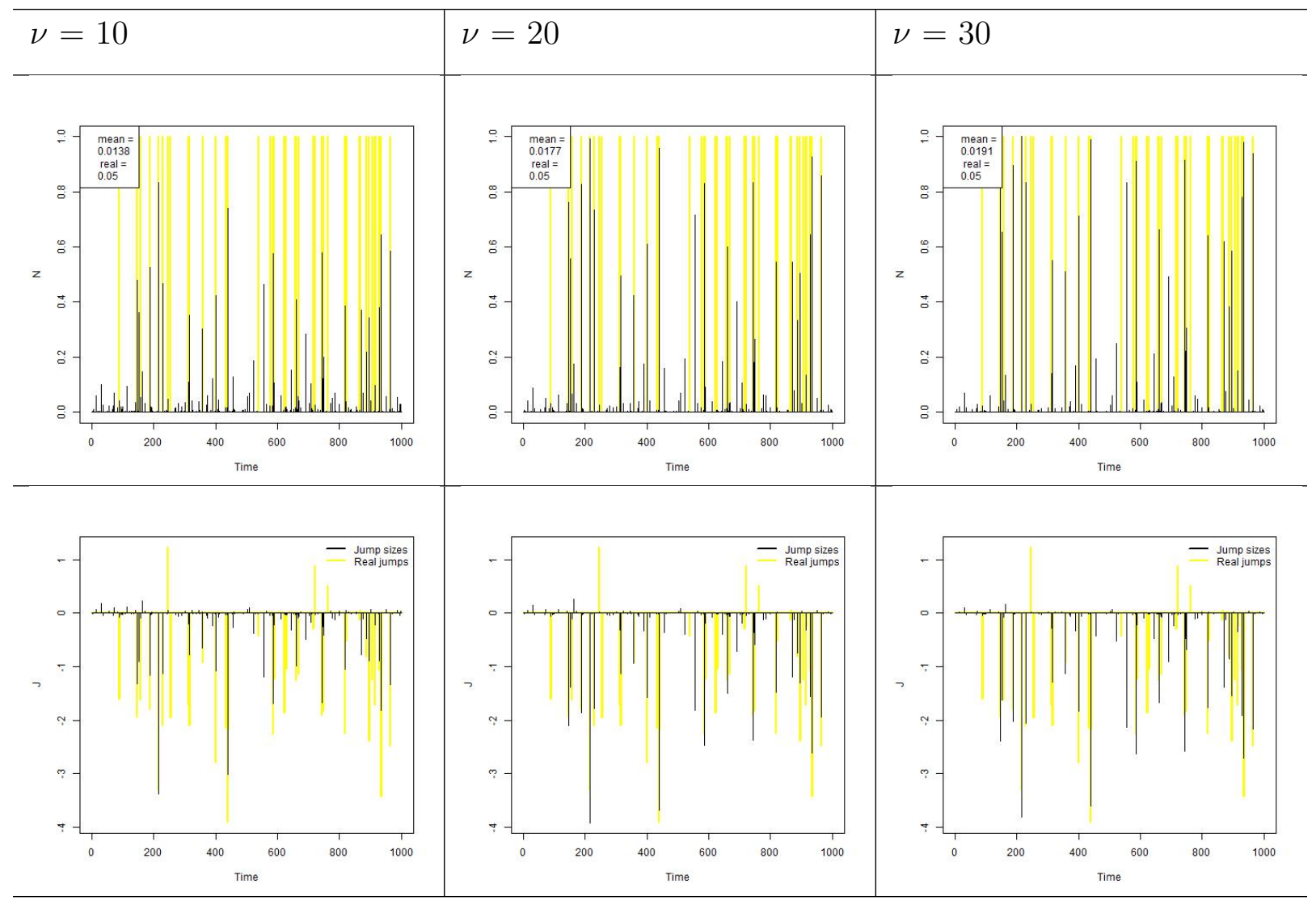

Figure 3.7: Simulation 4 - Weak Jumps - Jump probabilities and sizes

For weak jumps, Figure 3.7, it is evident that the model has difficult on separating jumps from distribution tail components. Since data is now generated from a distribution with heavier tail than Normal, this difficult is now exacerbated. Jump probabilities are underestimated in all cases. Also, it can be seen that only the strongest jumps were considered by the model as jumps, others were considered as part of distribution tail. 


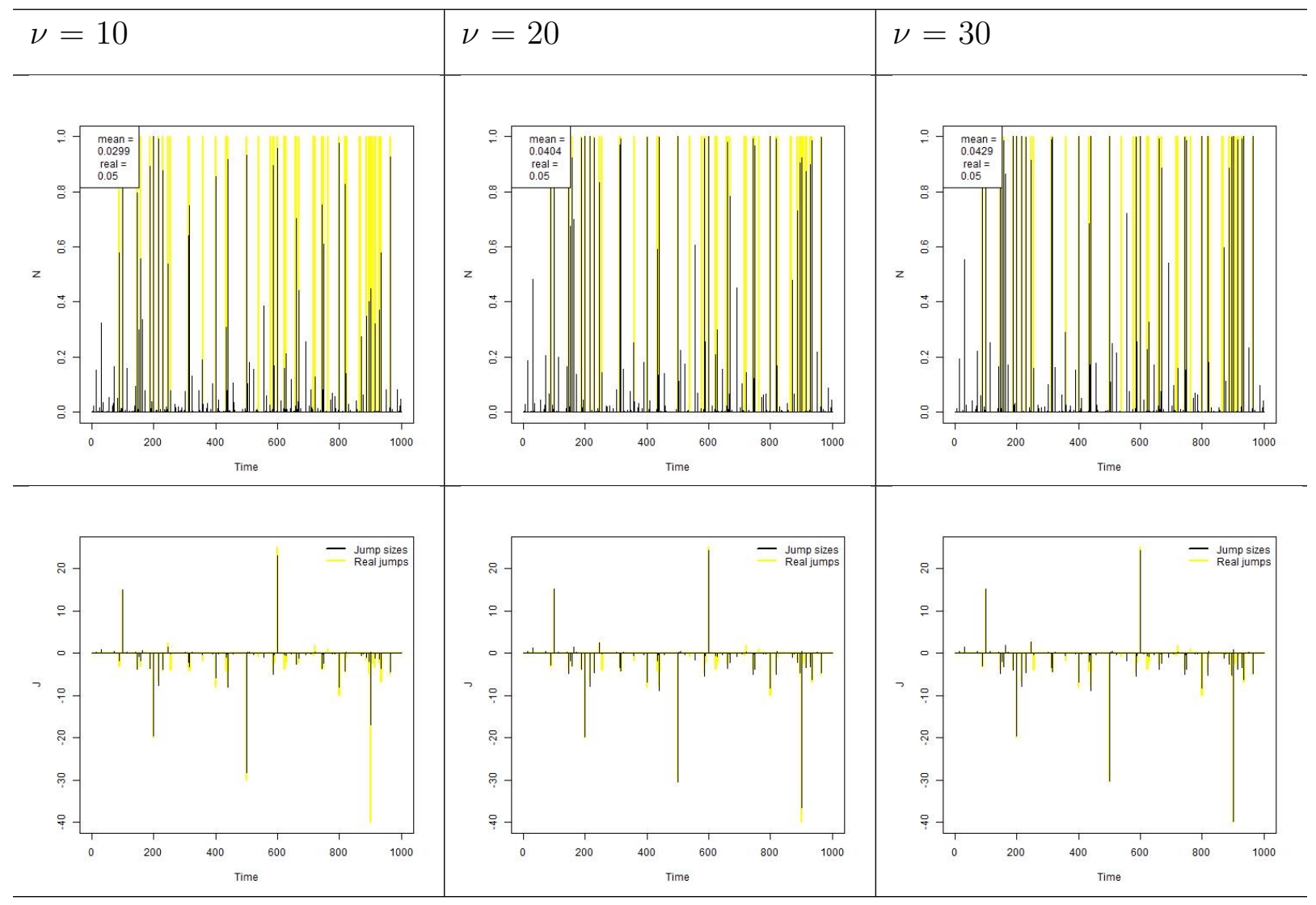

Figure 3.8: Simulation 4 - Manually Imputed Jumps - Jump probabilities and sizes

For manually imputed jumps, Figure 3.8, it can be seen that, for $\nu=10$ the model was not able to capture some of the jumps, since giving this much flexibility led to consider part of the jumps as a component of the distribution tail. The same is observed for $\nu=20$ on position 900, a small part of the jump size was left behind by the model. With $\nu=30$ the model was able to capture all manually imputed jumps precisely. 


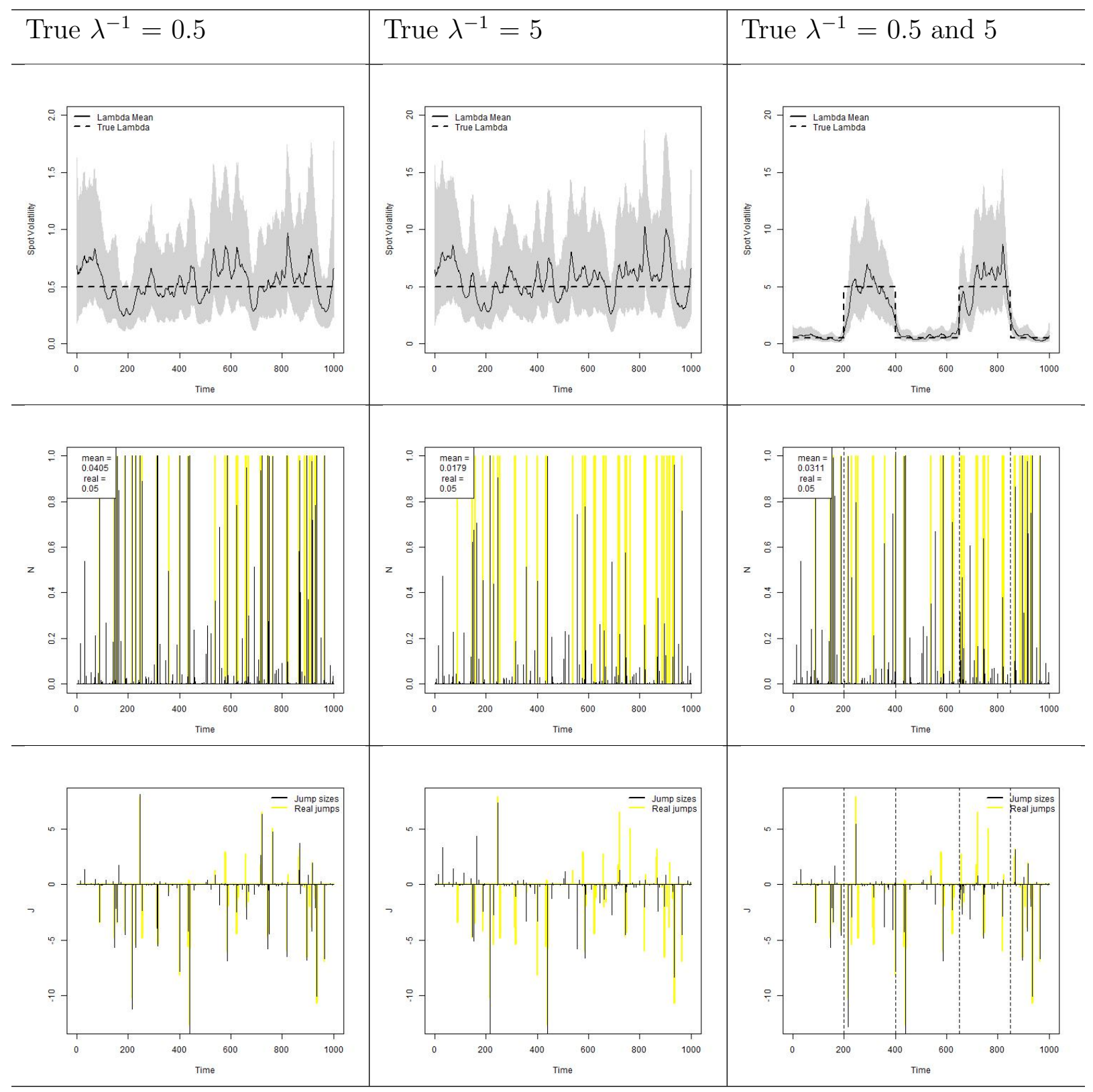

Figure 3.9: Simulation 5 - Influence of volatility changes

Figure 3.9 shows the effect of volatility changes on jump estimates. Continuous line represents the posterior mean and the gray area indicates the $95 \%$ credibility interval. Increases on estimated volatility affect the model increasing tail weight, since in the model $v_{t} \mid \gamma_{t} \sim N\left(0, \gamma_{t}^{-1} \lambda_{t}^{-1}\right)$. Therefore, some outliers can be captured by model tail, reducing observations that are considered jumps, 
affecting jump probabilities and size estimates. However, such effect was already expected, since in moments of market tensions, where risk rises above normal, excess in returns are not merely isolated speculative movements, that can be capture by the jump component, but reflex of the increase in market risk. It is notable that the model was able to capture the changes in volatility over the time, which is particularly important, since the main focus is on precisely estimating changes in volatility, which represents market risk.

\subsection{Simulation conclusions}

Based on simulations, it is possible to conclude that the model is able to capture both jump and heavy tails structures and estimate parameters with good precision. The model has some difficulty on estimating jump size variance, since estimation is based only observations which are considered jumps by the model, leading to a value generally higher than true parameter. It is also evident that when jumps are weak, the model has trouble on recognizing them, which leads to a drop on jump probabilities estimative and increase in jump sizes variance. This problem is worsen for higher threshold $\alpha$ values and smaller $\nu$ values.

Simulated data suggests that, for practical applications, where there is not much time and computational resources available and real parameters are not known, using $\alpha=0.70$ and $\nu=30$ would bring best results overall, but such parameters can be specified in other way by model user. If there is time and computational resources available, a sensibility analysis can be made for a grid of different $\alpha$ and $\nu$ values, as shown on the simulations, and best model can be chosen based on B-statistics.

Simulations also suggests that jumps are an effective way to capture isolated speculative movements in asset returns, so that the estimative of the risk is not influenced by such movements. When it comes to an increase on market volatility, jump role in the model is diminished, since excess in returns are, in this case, reflex of the increase in market risk. 


\section{Chapter 4}

\section{Applying model to stock market index data}

In this section the proposed model will be applied to stock market index data. The first dataset contains S\&P 500 stock index returns from January 2, 1980, to December 31, 1999. Excluding weekends and holidays, there are 5,054 daily observations for the S\&P. The second dataset contains iBovespa stock index returns from May 11, 2001, to December 18, 2015. Excluding weekends and holidays, there are 3,647 daily observations for iBovespa. Figures 4.1 and 4.2 shows a histogram for the indexes log returns. Table 4.1 provides summary statistic for the log returns, scaled by 100, Figure 4.3 shows S\&P500 log returns through time and Figure 4.4 shows iBovespa log returns through time. 


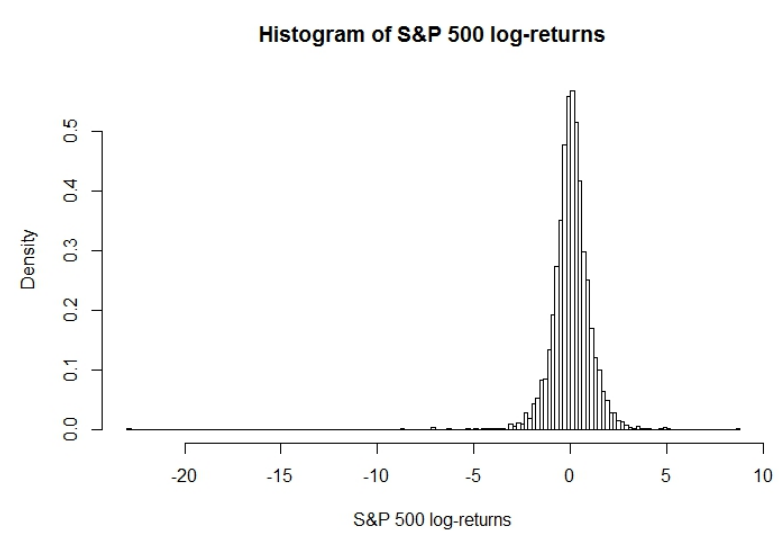

Figure 4.1: S\&P500 log return histogram

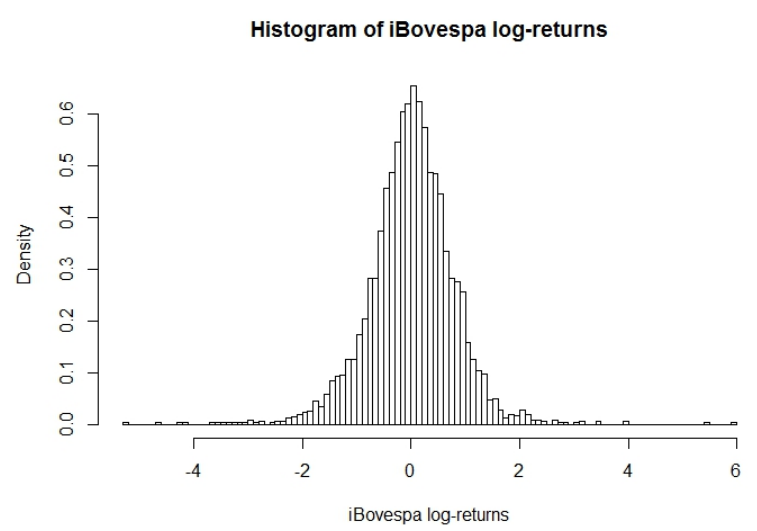

Figure 4.2: iBovespa log return histogram

\begin{tabular}{l||l|l|l|l|l|l}
\hline & Mean & Variance & Skewness & Kurtosis & Min & Max \\
\hline S\&P500 & 0.05205 & 0.9978 & -2.6357 & 63.0710 & -22.8997 & 8.7089 \\
\hline iBovespa & 0.0134 & 0.6137 & -0.0931 & 7.3176 & -5.2533 & 5.94038 \\
\hline
\end{tabular}

Table 4.1: Summary Statistics

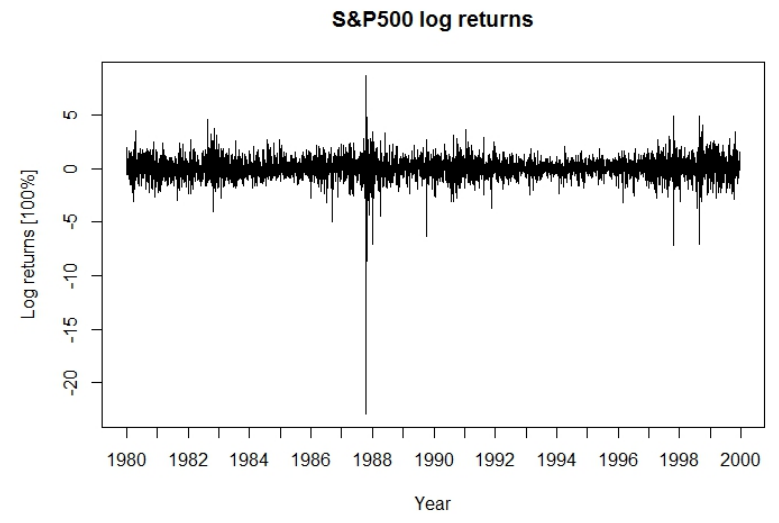

Figure 4.3: S\&P500 log return series

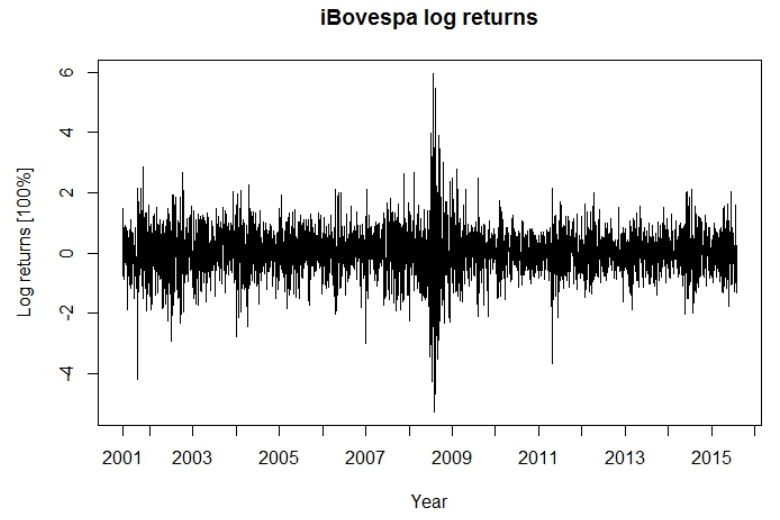

Figure 4.4: iBovespa log return series

As summary statistics, histogram and plot of time series suggests, the S\&P500 and iBovespa have different characteristics. For the analyzed period the american index has a much bigger amplitude on its daily returns than the brazilian 
index. Two main reasons for that are: the period being analyzed, since economy characteristics substantially changed from the decade of 80 to year 2000 and on; and also mechanisms of limiting loss that were recently implemented, in which assets trade are suspended in case of a big daily loss or gain. Due to such mechanisms, speculative movements are limited, avoiding big losses as the one observed in S\&P500 of $22.89 \%$ in one day. Due to that, it is expected that jumps will play a much greater role on american index than brazilian.

For applying on stock market index data, based on simulations conclusion that suggest $\nu=30$, a $\mathrm{G}(15,15)$ prior distribution was specified for $\gamma_{t}$ in order to obtain the Student's $t_{30}$-errors for the observation and system disturbances. The threshold, based on simulations conclusion, was fixed at $\alpha=0.7$ and the discount factor $\omega$ fixed at 0.9. All other priors and the MCMC parameters were specified in the same way it was for simulation, in section 3.2. MCMC results are shown on Appendix D.

\section{1 $\quad$ S\&P 500}

Table 4.2 provides parameter posterior means and standard deviation for the proposed model. $\overline{\lambda^{-1}}$ value represents mean and standard deviation for the set of $\lambda_{t}^{-1}$ means. Analogously, $\bar{\gamma}$ refers to the set of $\gamma$.

\begin{tabular}{l||l}
\hline Parameter & Estimated value \\
\hline$\mu$ & $0.0658(0.01066)$ \\
$\overline{\lambda^{-1}}$ & $2.3529(1.5823)$ \\
$\bar{\gamma}$ & $0.9997(0.0409)$ \\
$\rho_{y}$ & $0.0149(0.0022)$ \\
$\mu_{y}$ & $-0.3567(0.3895)$ \\
$\sigma_{y}$ & $2.6999(0.2906)$ \\
$\mathrm{B}$ & -1.8454 \\
\hline
\end{tabular}

Table 4.2: Parameter estimates for S\&P 500 index data 
Figure 4.5 provides jump sizes and probabilities for each observation. As observed on simulations, part of the excess in returns are absorbed by the distribution tail or in the form of an increase in variance, which would mean an increase on the risk measure. Before periods of higher volatility, it is possible to observe an increase in jump sizes and probabilities, when compared to periods with lower volatility, thus evidencing that such periods are preceded by speculative movements, captured in the model by jumps.

Figure 4.6 provides spot volatility estimates over the whole period, Jan/1980 to Dec/1999, and over two specific periods, 1987 to 1989, when took place the Black Monday, on Oct/1987, and 1997 to 1999, when Asian and Russian financial crisis shaken the stock market. Continuous line represents the posterior mean and the gray area indicates the $95 \%$ credibility interval for spot volatility. Although measure differs, since a different distribution specification was made, volatility behavior is consistent with Eraker et al. (2003) [9] findings, with peaks occurring at the same time.

Figure 4.7 provides posterior mean for mixture component $\gamma$. As it gets farer from 1, it indicates increasing activity on capturing heavy tails in returns. A distinguishable point is seen on Oct/1987, when Black Monday occurred. The model consider this point as a heavy tail event, thus reducing jump size on that point and increasing volatility measure. Variance, Figure 4.8, defined as $\gamma_{t}^{-1} \lambda_{t}^{-1}$ shows behavior similar to volatility. 

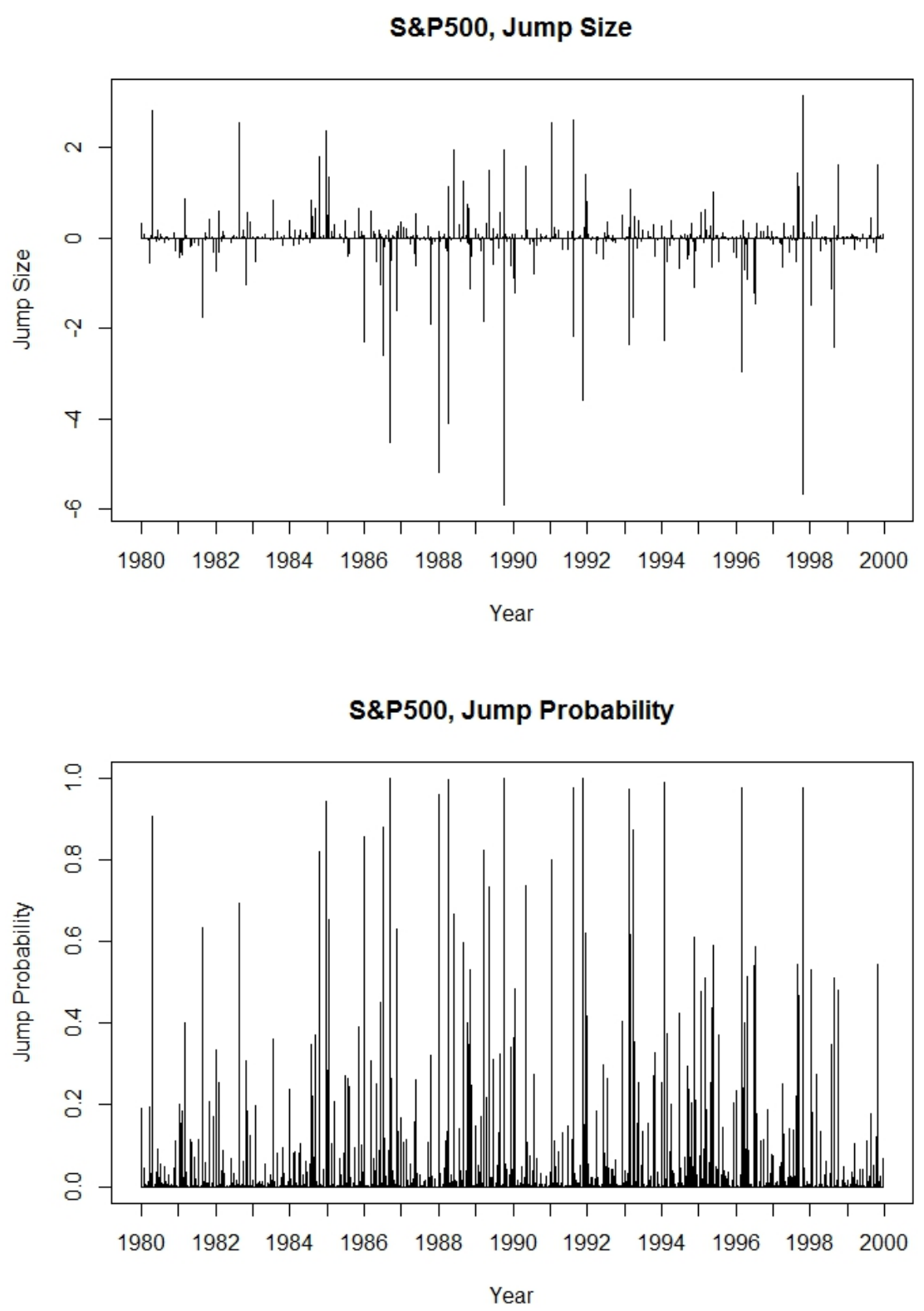

Figure 4.5: Estimated jump times and sizes for the S\&P500 index 

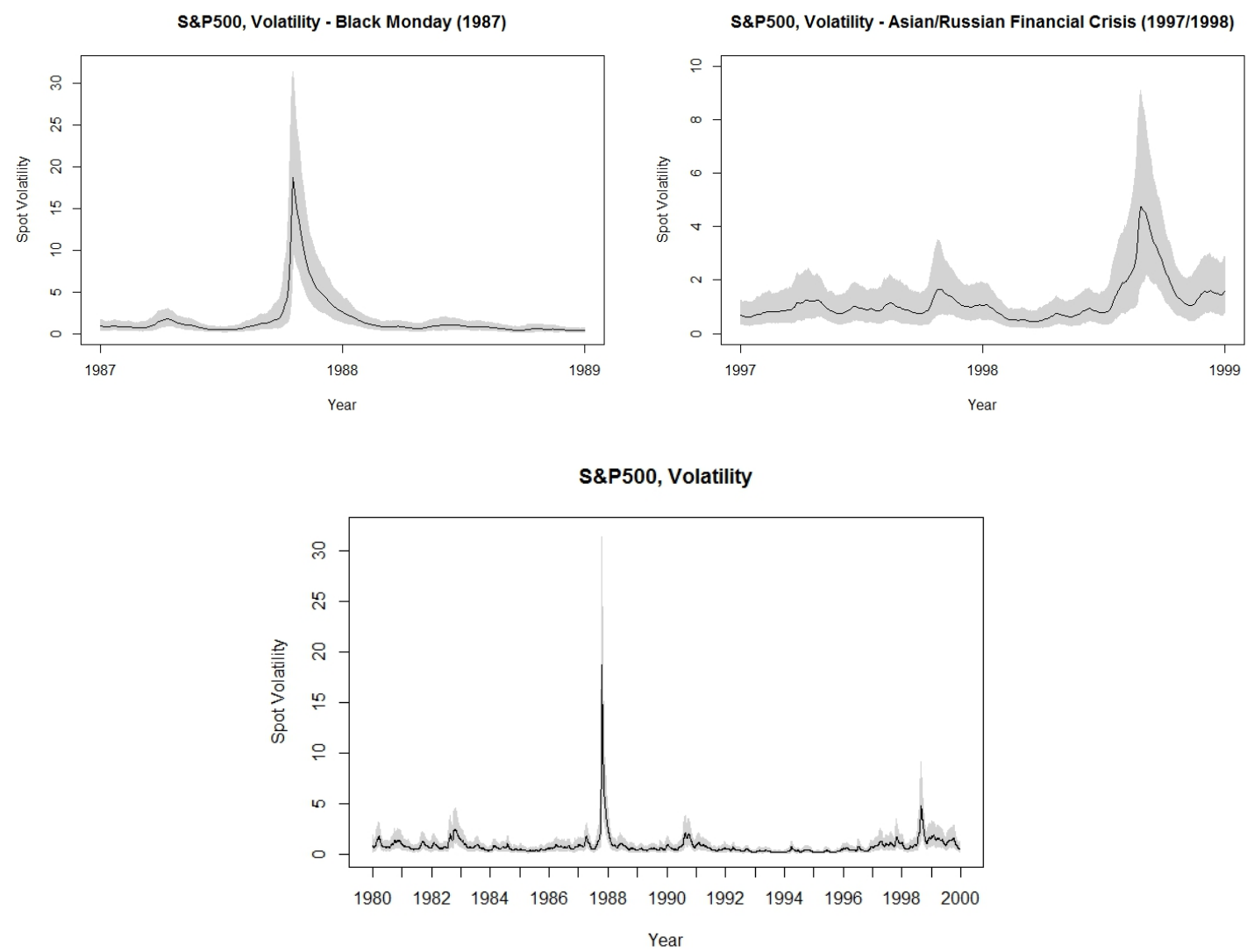

Figure 4.6: Estimated volatility for the S\&P500 index. Continuous line represents the posterior mean. The grey area indicates the $95 \%$ credibility intervals.

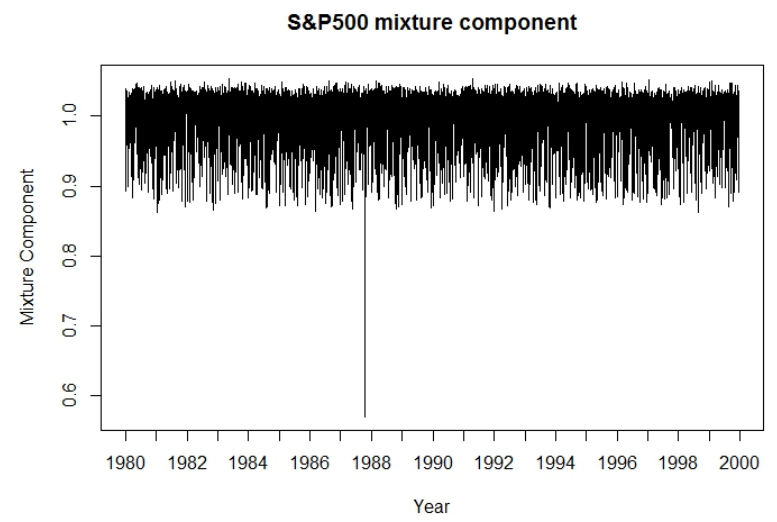

Figure 4.7: Mixture component, $\gamma_{t}$

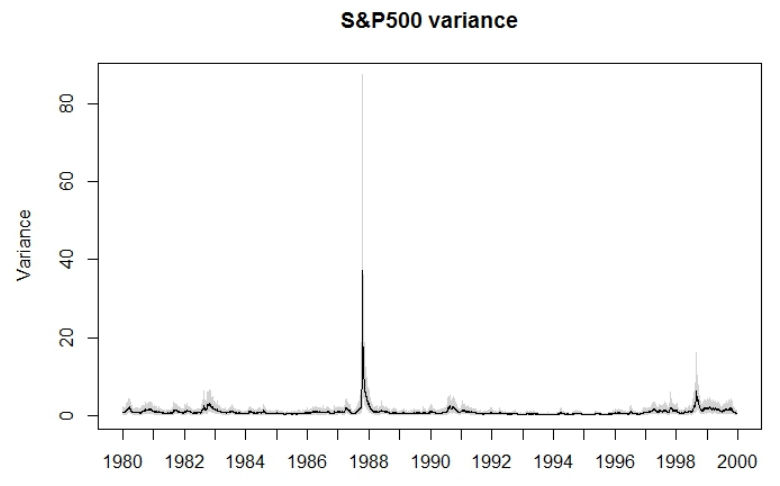

Year

Figure 4.8: Variance, $\gamma_{t}^{-1} \lambda_{t}^{-1}$ 


\section{2 iBovespa}

Table 4.3 provides parameter posterior means and standard deviation for the proposed model. $\overline{\lambda^{-1}}$ value represents mean and standard deviation for the set of $\lambda_{t}^{-1}$ means. Analogously, $\bar{\gamma}$ refers to the set of $\gamma$.

\begin{tabular}{l||l}
\hline Parameter & Estimated value \\
\hline$\mu$ & $0.0300(0.0107)$ \\
$\overline{\lambda^{-1}}$ & $0.5655(0.6741)$ \\
$\bar{\gamma}$ & $0.9997(0.0389)$ \\
$\rho_{y}$ & $0.0090(0.0021)$ \\
$\mu_{y}$ & $-0.3025(0.4947)$ \\
$\sigma_{y}$ & $1.9987(0.3357)$ \\
$\mathrm{B}$ & -1.6640 \\
\hline
\end{tabular}

Table 4.3: Parameter estimates for iBovespa index data

Figure 4.9 provides jump sizes and probabilities for each observation. Since iBovespa returns on this period is more stable when compared to S\&P 500 index on 1987 to 1999 period, jump sizes and probabilities are smaller than what is observed in American index. Since log return magnitudes are smaller than S\&P's, model heavy tails tend to accomodate more observations, which also reduces jump probability and size estimates. In iBovespa index, increase on jumps probabilities and sizes can also be observed days before increases on volatility, so that the presence of higher jumps can indicate near future increases on market risk.

Figure 4.10 provides the spot volatility estimates over the whole period, May/2001 to Dec/2015, and over two specific periods, 2007 to 2009, when took place the American subprime crisis, and 2014 to early 2015, when Brazilian presidential elections brought instability to stock markets. Continuous line represents the posterior mean and the gray area indicates the $95 \%$ credibility interval for spot volatility. Increase in volatility is consistent with political or financial events that occurred on the period. 
Figure 4.11 provides posterior mean for mixture component $\gamma_{t}$. As it gets farer from 1, it indicates activity on capturing heavy tails in returns. It's possible to see that this component is persistently capturing heavy tails, which can explain the absence of stronger jumps for this market. Variance (Figure 4.12) defined as $\gamma_{t}^{-1} \lambda_{t}^{-1}$ shows behavior similar to volatility. 
iBovespa, Jump Size

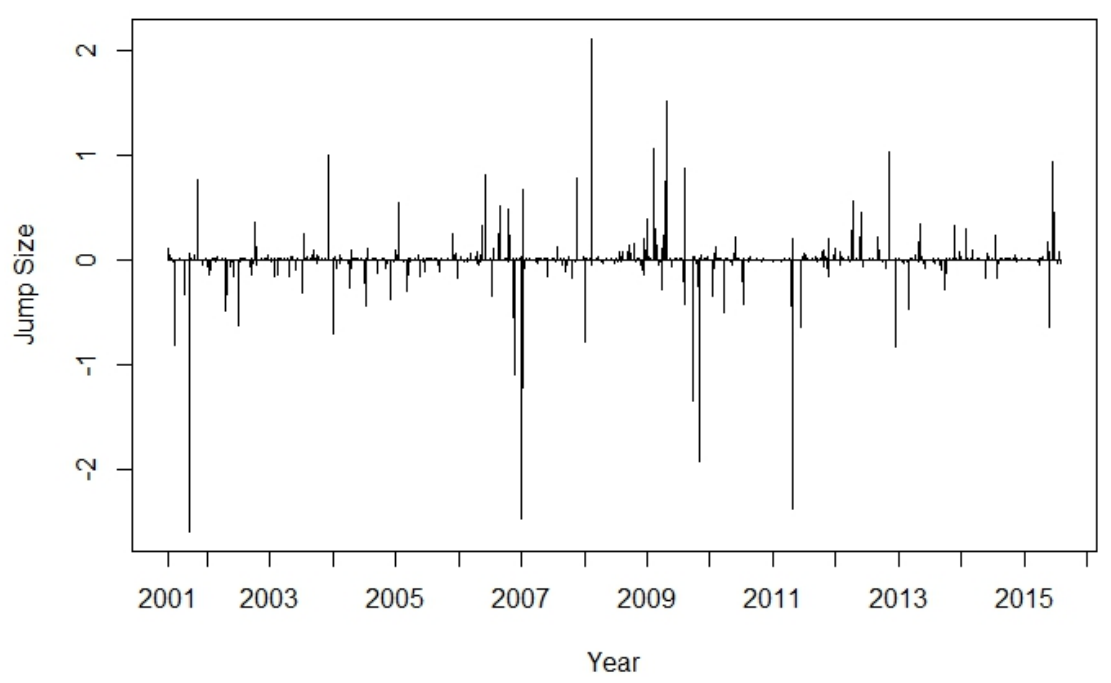

iBovespa, Jump Probability

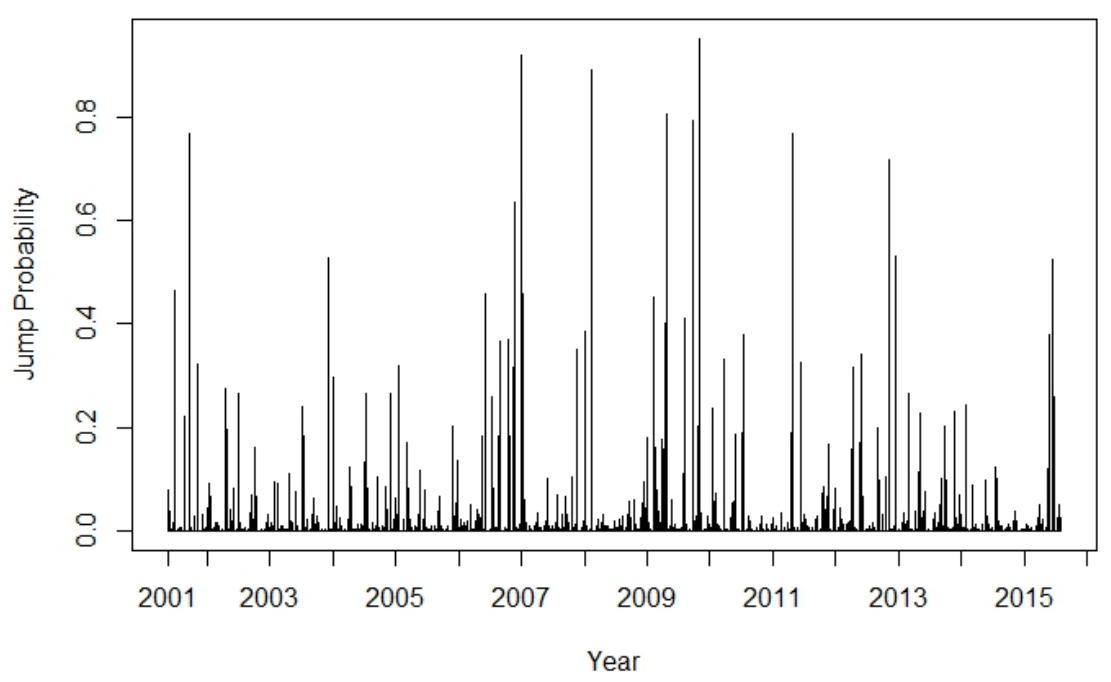

Figure 4.9: Estimated jump times and sizes for the iBovespa index 

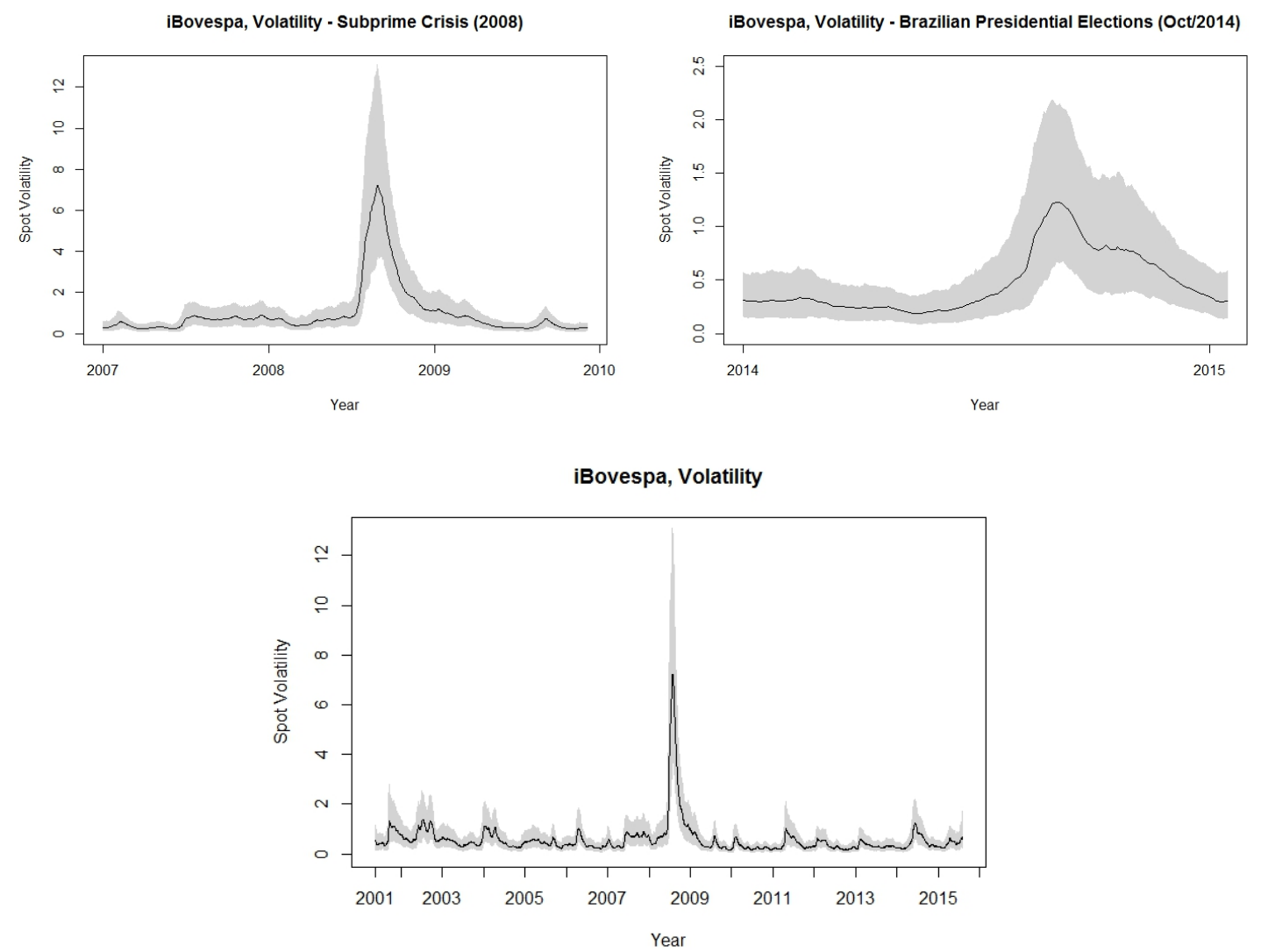

Figure 4.10: Estimated volatility for the iBovespa index. Continuous line represents the posterior mean. The grey area indicates the $95 \%$ credibility intervals.

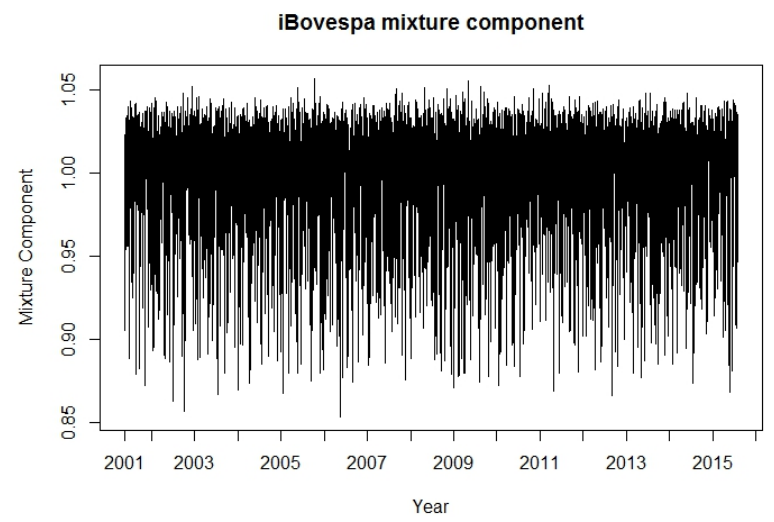

Figure 4.11: Mixture component, $\gamma_{t}$

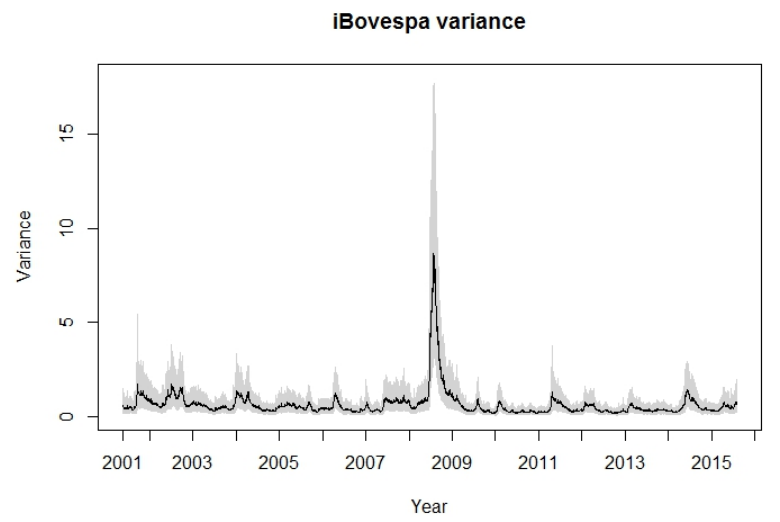

Figure 4.12: Variance, $\gamma_{t}^{-1} \lambda_{t}^{-1}$ 


\subsection{Experimental conclusions}

The NGSVJ model was able to capture speculative movements in the market through the jump components and detect periods with increased market risk through the volatility component. Results obtained by applying the model to S\&P 500 return series are consistent with Eraker et al. (2003) [9] findings, since volatility peaks occur at same times. Historical events of known volatility effects on financial markets, such as Black Monday and Asian/Russian Financial Crisis, for S\&P index, and Subprime crisis and Presidential Elections, for iBovespa index, can be detected by the model precisely.

Results also suggest that the model can adapt to data despite the characteristics of the market being analysed. Jumps play a greater role on markets with bigger discrepancy between returns, like in S\&P index, where log returns amplitude is around 31 points, three times bigger than iBovespa's log returns amplited of just around 11 points. On iBovespa index, less jumps are detected and some of excess returns are absorbed by distribution tail.

One recurrent behavior, that is also noted by Eraker et al. (2003) [9], is the increase on jump probabilities and sizes days before increases in volatility. This pattern can be observed on both indexes and may indicate that jumps, beyond being a tool to capture speculative movements, can be a way of forecasting near future increases on market risk. 


\section{Chapter 5}

\section{A proposal for weak jumps scenario}

This chapter presents an alternative method for jump detection and simulations on using such method.

\subsection{Jump Detection Algorithm: a Non-Parametric Approach}

On trying to solve the issue of estimating jumps on a weak jumps scenario, an alternative jump detection algorithm based on a non parametric approach can be used. For that purpose, the algorithm proposed by Riley (2008) [21] can be used. The Sequential Average method consists on comparing the difference between the average values within a pair of adjacent windows of adjustable length against a selectable jump threshold. If a potential jump is detected, the following points are examined in an averaging window to accept or reject the jump. According to Riley (2008) [21], the advantage of this method is that it gives the right jump location and no offset parameter is needed. A similar approach is also developed by Lee 
and Maykland (2008) [14]. If the length for analysis is 1, the algorithm for the process is given by the following chart.

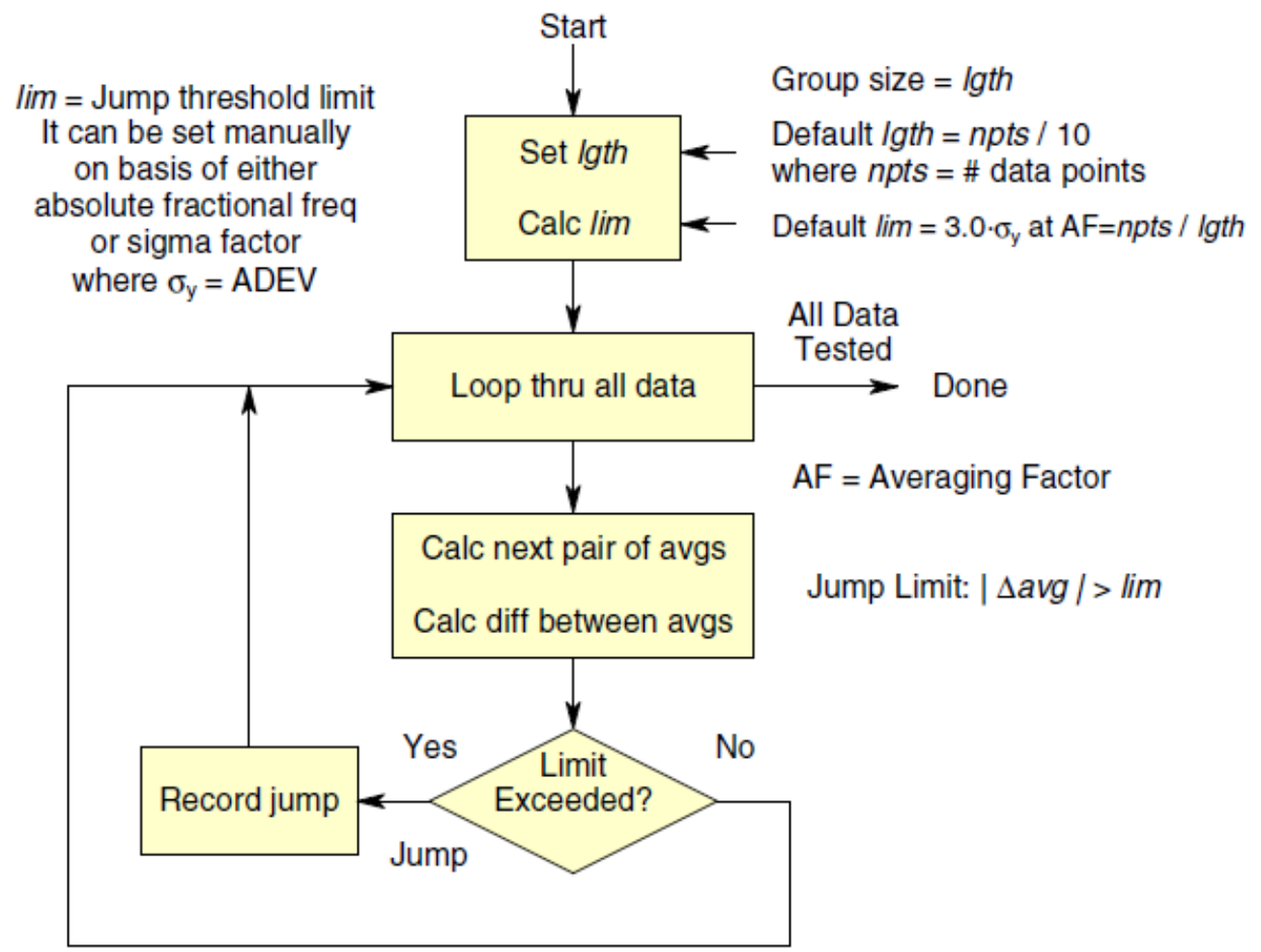

Figure 5.1: Block average jump detection flowchart. Source: Riley(2008).

In other words, if the observation $y_{t+1}$ is out of the interval $y_{t} \pm Z \times$ $\sqrt{\gamma_{t}^{-1} \lambda^{-1}}$, where $Z$ is a threshold selected according to the area of a Normal curve corresponding to a tail probability ${ }^{1}$, it is considered a jump at time $t+1$.

The jump probabilities can be easily calculated by getting the average value of $J_{t}$. The average in this case would be the number of times there was considered a jump at time $\mathrm{t}$ divided by the number of iterations taken, which would be a number inside $[0,1]$.

\footnotetext{
${ }^{1}$ Based on a $\mathrm{N}(0,1)$ test on which $\mathrm{Z}$ refers to the value in which probability of occurrence is low enough to be considered a jump.
} 


\subsection{Simulations}

For the non-parametric approach, the simulation data used was the same as Simulation 4 - Student's t distribution with 20 degrees of freedom and weak jumps. For a $Z$ selected so that the cut-point is $95 \%$ and $99 \%$ of a Normal curve, three different values for $\nu$ were tested: 10, 20 and 30 .

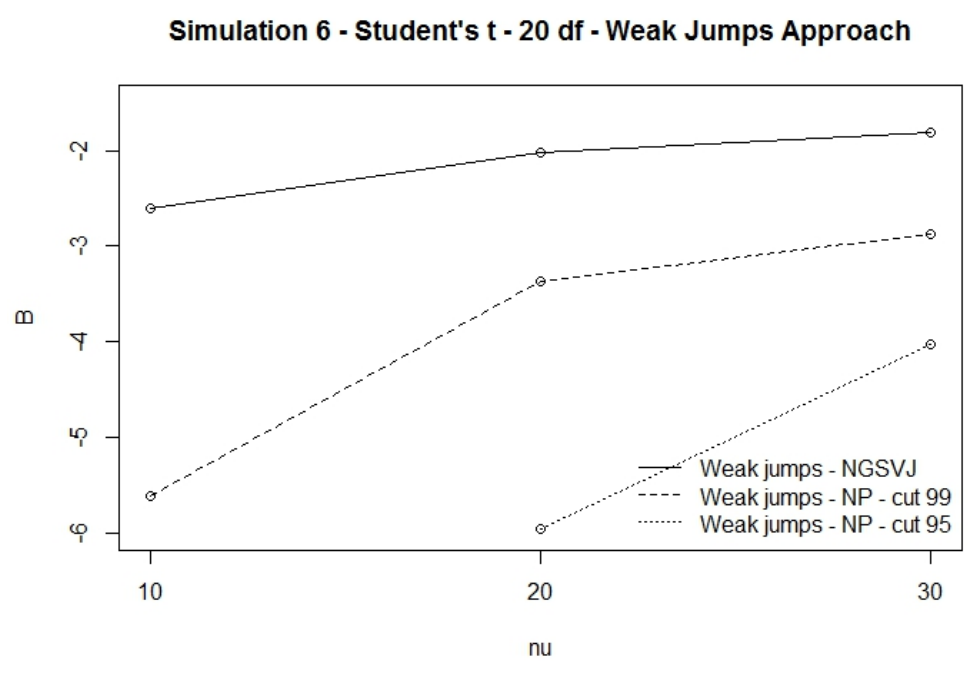

Figure 5.2: B-statistics comparison for Student's t scenario - Non Parametric Approach

Figure 5.2 shows B-satistics results for the parametric (NGSVJ) approach, obtained on Simulation 4, and non-parametric (NP). In all cases, the parametric approach had a better fit according to B-statistic. 


\begin{tabular}{l||l|l|l|l}
\hline \multicolumn{5}{c}{ Weak Jumps - NGSVJ } \\
\hline Parameters & True & $\nu=10$ & $\nu=20$ & $\nu=30$ \\
\hline$\mu$ & 0.10 & $0.0491(0.0300)$ & $0.0603(0.0245)$ & $0.0681(0.0245)$ \\
$\lambda^{-1}$ & 0.5 & $0.5385(0.1371)$ & $0.5534(0.1466)$ & $0.5592(0.1507)$ \\
$\gamma^{-1}$ & & $1.0019(0.1091)$ & $1.0000(0.0592)$ & $0.9998(0.0412)$ \\
$\rho_{y}$ & 0.05 & $0.0138(0.0038)$ & $0.0177(0.0042)$ & $0.0191(0.0044)$ \\
$\mu_{y}$ & -1.5 & $-2.1209(0.6937)$ & $-2.3737(0.4834)$ & $-2.4673(0.3964)$ \\
$\sigma_{y}$ & 1 & $3.176(4.2308)$ & $1.7956(1.7863)$ & $1.1639(1.2341)$ \\
$\mathrm{B}$ & & -2.6069 & -2.0139 & -1.8102 \\
\hline
\end{tabular}

Weak Jumps - Non Parametric Approach - cut 99\%

\begin{tabular}{l||l|l|l|l}
\hline Parameters & True & $\nu=10$ & $\nu=20$ & $\nu=30$ \\
\hline$\mu$ & 0.10 & $0.0711(0.0391)$ & $0.0692(0.0331)$ & $0.0706(0.0349)$ \\
$\lambda^{-1}$ & 0.5 & $0.4866(0.1087)$ & $0.5100(0.1149)$ & $0.5163(0.1122)$ \\
$\gamma^{-1}$ & & $1.0019(0.0979)$ & $0.9986(0.0555)$ & $0.9993(0.0384)$ \\
$\rho_{y}$ & 0.05 & $0.0483(0.0066)$ & $0.0453(0.0064)$ & $0.0453(0.0064)$ \\
$\mu_{y}$ & -1.5 & $-1.9811(1.7849)$ & $-1.9665(1.3807)$ & $-1.8711(1.2589)$ \\
$\sigma_{y}$ & 1 & $2584.51(77880.84)$ & $2.7826(6.0222)$ & $2.4737(1.1679)$ \\
$\mathrm{B}$ & & -5.6193 & -3.3669 & -2.8792 \\
\hline
\end{tabular}

Weak Jumps - Non Parametric Approach - cut 95\%

\begin{tabular}{l||l|l|l|l}
\hline Parameters & True & $\nu=10$ & $\nu=20$ & $\nu=30$ \\
\hline$\mu$ & 0.10 & $0.086(0.0363)$ & $0.0814(0.0301)$ & $0.0817(0.0278)$ \\
$\lambda^{-1}$ & 0.5 & $0.3983(0.0811)$ & $0.3996(0.0794)$ & $0.4022(0.0791)$ \\
$\gamma^{-1}$ & & $0.9986(0.0873)$ & $0.9963(0.0489)$ & $0.9966(0.0342)$ \\
$\rho_{y}$ & 0.05 & $0.1168(0.0098)$ & $0.1113(0.0096)$ & $0.1145(0.0098)$ \\
$\mu_{y}$ & -1.5 & $-1.0547(1.1567)$ & $-0.9757(0.7974)$ & $-0.9108(0.6494)$ \\
$\sigma_{y}$ & 1 & $1.9908(0.645)$ & $2.0355(0.5762)$ & $1.9908(0.645)$ \\
$\mathrm{B}$ & & $-\infty$ & -5.9567 & -4.0273 \\
\hline
\end{tabular}

Table 5.1: Simulation 6 - Non-Parametric Approach - Student's t - 20 degrees of freedom

For non-parametric approach, there is no significant change on jumps estimation by varying $\nu$ parameter. Since this approach is based on critical regions of the distribution, as tail get heavier, smaller is the critical region, so the num- 
ber of outliers stays more or less the same. For cut-point $=99 \%$ and $\nu=30$, non-parametric approach shows point estimates slightly closer to real values than other models in general. However, B-statistics indicates that model fit is worse than NGSVJ parametric approach. Figure 5.3 gives a glue of the reason why this happens: even though point estimate matches with real value, model is not able to capture precisely jump times, underestimating probabilities for real jump times and detecting jumps where there are not.

Using cut-point $=95 \%$, the model over-estimates jump probabilities. This leads to worse B-satistics when compared to other models.

Figure 5.3 compares the three models, each of them with parameters that lead to the highest B-statistics possible. Even though non-parametric approach can capture more jumps, it misses several points, so that jump estimates may not reflect real values. Non-parametric model captures non existing jumps several times, and also misses real jumps. There are several cases of wrong classifications, which can be seen on jump sizes graphics. 


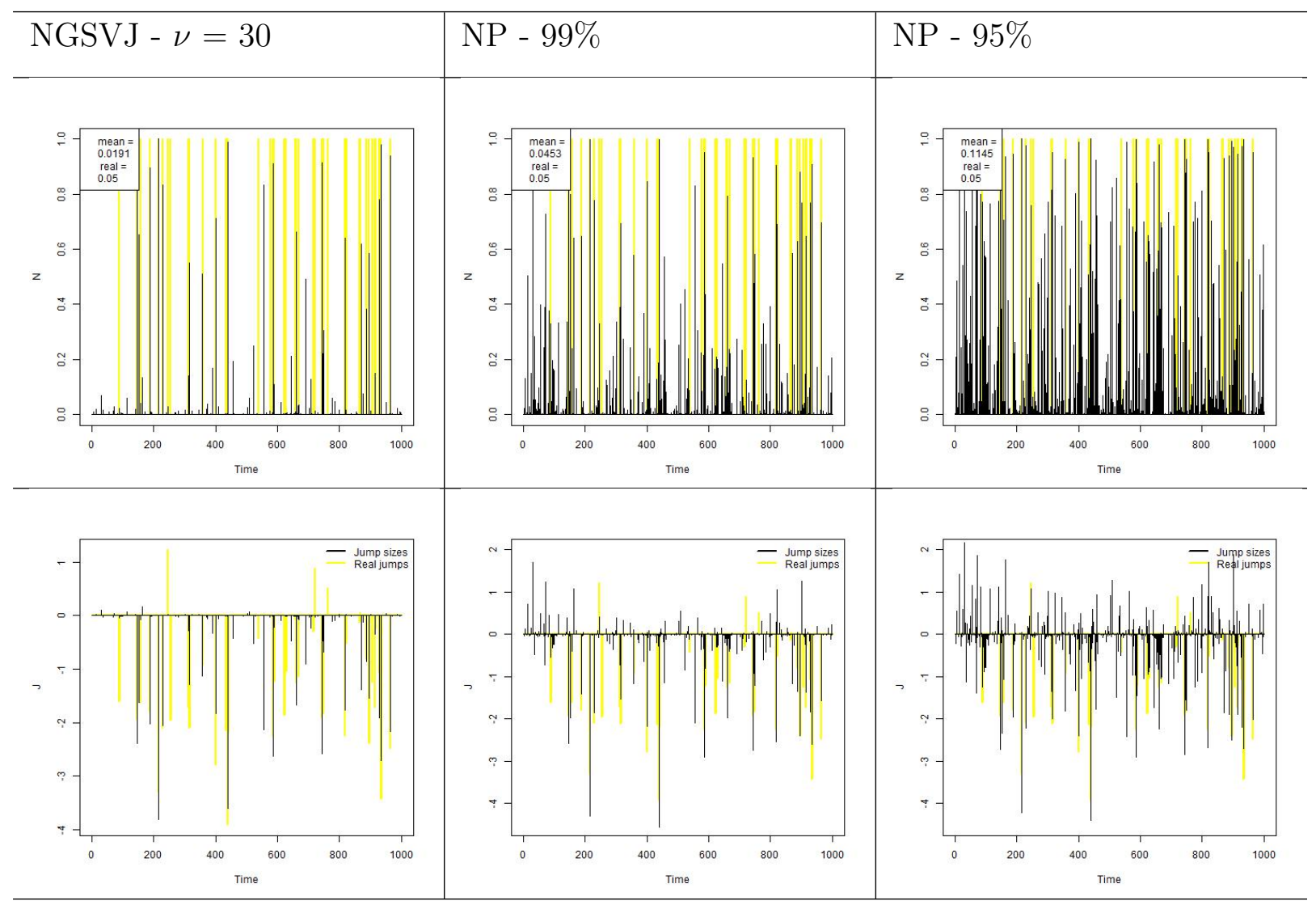

Figure 5.3: Simulation 6 - Weak Jumps - Non-Parametric Approach

\subsubsection{Simulation conclusions}

The non-parametric approach was not effective on estimating jump times and sizes. This affects estimation of other parameters, and has a negative effect on the model as a whole. Even tough non parametric approach could get better point estimates in some cases, in a practical application it can miss real values, since data is generally more complex and the jump miss-classifications can interfere on other parameters estimates. 


\section{Chapter 6}

\section{Discussion about the NGSVJ model}

There are a lot of works on stochastic volatility models for univariate financial time series. This work used as base for comparison the models presented by Eraker et. al. (2003) [9], but there are also developments on SV models, as the proposed by Warty et al (2014) [26], using variance-gamma jumps, and Nakajima and Omori (2007) [18], including in the model the leverage effect together with heavy tails and correlated jumps.

Since a lot has been already discussed on these previous works, one might ask what is the gain on continue developing methods to model volatility for univariate financial time series. This chapter focus on discussing the key points that justify the importance of NGSVJ model as an alternative to the classic SV model and its variations.

\subsection{NGSVJ model advantages}

The most notable advantage of using NGSVJ is its computational simplicity 
and the structure of the DLM model, on which it is based, that grants an automatic sampling process for parameters.

Using Gibbs Sampler to obtain a sample from conditional posterior distributions is computationally cheaper than recurring to Metropolis based algorithms. Since Metropolis is an accept-reject algorithm, it can take several steps until a full sample is obtained in order to make statistical inference, unless a very good proposal distribution is given to the algorithm. On using Gibbs Sampler, only one step is needed in order to obtain the same sample. This is specially relevant when dealing with high dimensional data, such as market daily returns from several years.

Due to this computational simplicity, NGSVJ model can be run on an ordinary home computer and give reliable results for analysis in some hours, even for long iteration chains, such as 100,000 observations. The practical consequence of such advantage is that the model can be run after market closes and results will be available before market opens on the next day. Also, it can potentially bring cost reductions and be available for any market player, since there is no need for advanced hardware configuration in order to the computer to run the model.

As the model is build over the Dynamic Linear Model with scale mixtures proposed by Gamerman et al.(2013) [10], it takes advantage of the sampling process mentioned on Chapter 2.4.1. Theorems 1 and 2 cited by Gamerman et al. (2013) [10] can be applied so that an exact sample of Volatility parameter $\lambda_{t}^{-1}$ is obtained. The two main advantages that comes from this process are: there is no need to make approximations in order to estimate volatility, and it is a faster method, since all parameters are sampled at once.

Another advantage is the model flexibility. It can be adapted to include jumps, covariates, heavy tails and different distributions can be adopted based on the mixture component used. In this work, Student-t distribution was used trough a Gamma distributed mix component, but other distributions can also be used to give satisfactory results. 


\subsection{Comparison to other models}

Since SV models presented by Eraker et al. (2003) [9] have a different structure from the proposed model, comparison between models is not straightforward. Comparison can be made between parameters that exist on both NGSVJ and SV models with the same roles on both models, that is, equilibrium return and jump related parameters. Table 6.1 compares posterior mean of common parameters estimates between the proposed model and the stochastic volatility with jumps on returns (SVJ), with independently arriving jumps on returns and volatility (SVIJ) and contemporaneous arriving jumps (SVCJ), omitting the ones that are exclusive for each model, for S\&P 500 returns.

\begin{tabular}{l||l|l|l|l}
\hline & NGSVJ & SVJ & SVCJ & SVIJ \\
\hline$\mu$ & $0.0658(0.01066)$ & $0.0496(0.0109)$ & $0.0554(0.0112)$ & $0.0506(0.0111)$ \\
$\rho_{y}$ & $0.0149(0.0022)$ & $0.0060(0.0021)$ & $0.0066(0.0020)$ & $0.0046(0.0020)$ \\
$\mu_{y}$ & $-0.3567(0.3895)$ & $-2.5862(1.3034)$ & $-1.7533(1.5566)$ & $-3.0851(3.2485)$ \\
$\sigma_{y}$ & $2.6999(0.2906)$ & $4.0720(1.7210)$ & $2.8864(0.5679)$ & $2.9890(0.7486)$ \\
\hline
\end{tabular}

Table 6.1: Comparison between NGSVJ model and SVJ models in Eraker et. al (2003) [9] for S\&P 500 returns

NGSVJ model captures more jump points than Eraker et al. (2003) [9] SV models. This leads to a smaller estimative for $\mu_{y}$, since more points are captured as jumps. It also identify volatility peaks on same periods that Eraker et al. (2003) [9] , on Black Monday (1987) and Asian/Russian financial crisis (1997/1998), as can be seen in Figure 6.1. Even though volatility magnitude is not the same for both models, which is plausible, since model specification differs, using any of both volatility estimatives will lead to same conclusions for practical applications. 

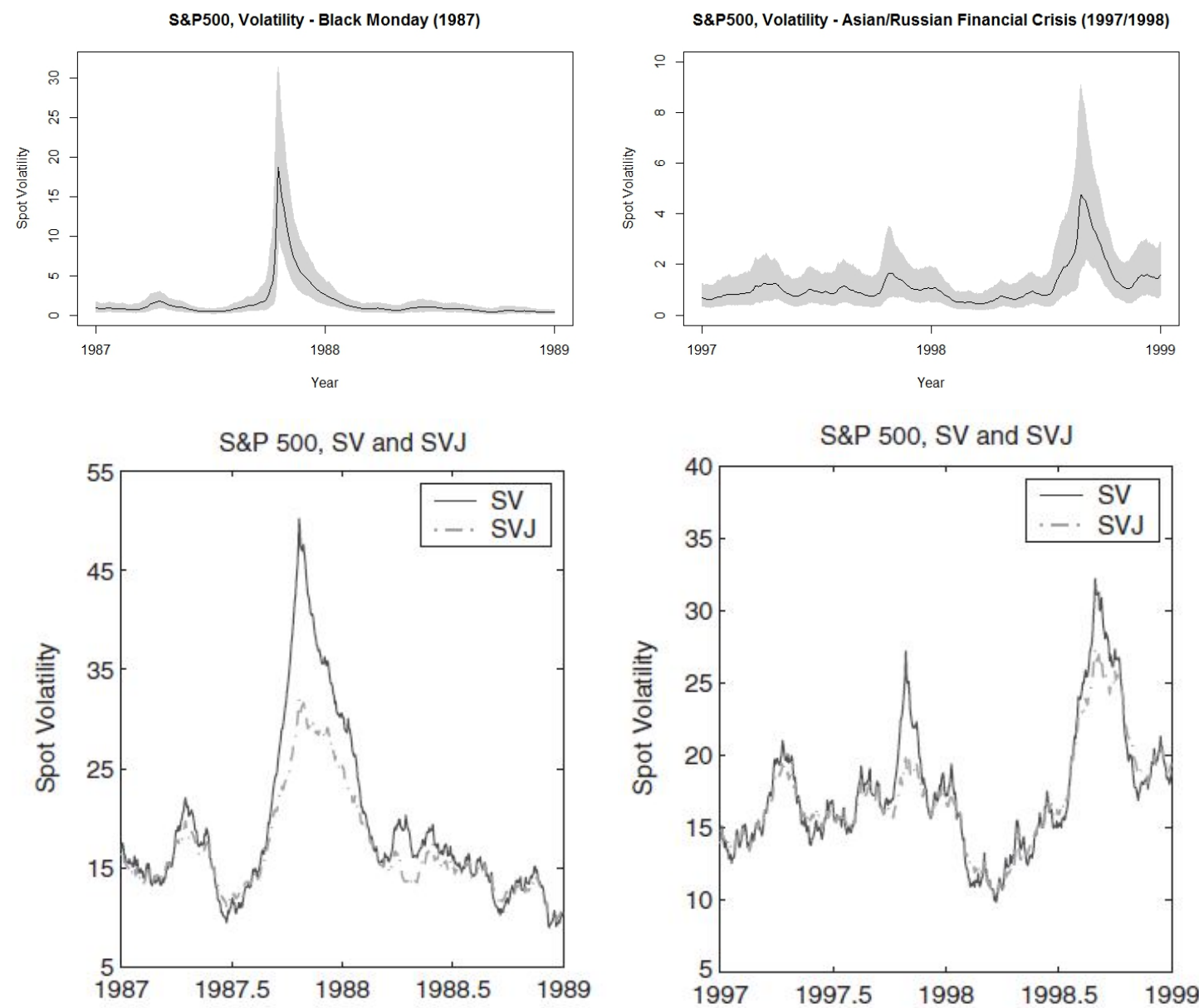

Figure 6.1: Comparison between estimated volatility on NGSVJ model and Eraker et al. (2003) [9] models. 


\section{Chapter 7}

\section{Conclusion}

The proposed model has a much more simple structure to deal with the inclusion of heavy tails and jumps than the other methods commonly used. The possibility of using only Gibbs Sampling method to sample from the posterior distributions, together with the block sampling structure, give efficiency and speed to the model, which is crucial in day-to-day operations.

The inclusion of jumps in the model reduces substantially the volatility estimate. This would have several impacts on risk analysis, since less volatility indicates less risk, in other words, knowing that some event was a jump, or tail event, and not a recurrent market event would mean that such asset is still a safe bet. Usually an increase of jump frequency is observed near the occurrences of market anomalies, such as Black Monday and the Asian and Russian financial crisis, in S\&P 500, and 2008 crisis and Brazilian presidential elections, in iBovespa, which may indicate that it can also be used for predicting near future increases on market risk.

The easiness to include mixture variables in the model is another advantage. By doing that, there are no simplifications of reality by assuming Gaussian distribution, since it is well documented on the literature that financial asset returns data have heavy tailed distribution.

Two main difficulties showed up during the development of the model: the 
first is due to dealing with high dimensional data, to keep up speed and save memory in the computer, optimizations must be done in the code in order to be able to run it. Such optimizations include: block sampling, squeezing the number of variables declared and the dimensionality of such variables, creating a burn-lags routine such that it only saves values that are in fact relevant; the second is on estimating jumps when jump sizes are small, since the model struggles to detect jumps that are inside distribution tail, which is worsen by using a heavy tailed distribution.

The attempt of using a non-parametric method, based on a normal curve tail analysis, such as proposed by Riley (2008) [21], for detecting jumps in a weak jumps scenario was not effective, since there is a lot of miss-classification using such method, which causes more harm than good to the model.

For day-to-day operations, the automatic model is effective and can be used in order to estimate market volatility, which can be used for options pricing, VaR calculations, measuring risk, etc. Its lower computational cost compared to Metropolis-based algorithms make it accessible to firms and investors who does not have access to high technology computers - common personal computers are able to run the model and bring reliable results in some hours. Using an Intel Core i5 $2310 \mathrm{CPU}$ at $2.90 \mathrm{Ghz}, 4 \mathrm{~GB}$ RAM, and 64 bit Windows Seven operating system, it took approximately 7 hours to get results from S\&P 500 index and 5 hours and a half to get results from iBovespa index.

For future works is intended to extend the model to the multivariate case, where, instead of analysing one asset individually, an asset portfolio risk is analysed as a whole. Another extension intended is the inclusion of jumps in volatility, as suggested by Eraker et al. (2003) [9], without having to appeal to Metropolis based algorithms, in order to keep the model fast and accessible. Some other possibilities include working with a skew heavy tailed distribution, as in Nakajima and Omori (2007) [17], or working with a stationary evolution to log-volatility, but still maintaining the block sampling, so that it allows to capture the leverage effect and correlations between mean and volatility. 


\section{Bibliography}

[1] Abanto-Valle, C. Lachos, V. and Dey, D. (2015). Bayesian Estimation of a Skew-t Stochastic Volatility Model. Methodology and Computing in Applied Probability, 17:721-738.

[2] Bollerslev, T. Gibson, M. and Zhou, H. (2010). Dynamic estimation of volatility risk premia and investor risk aversion from option-implied and realized volatilities. Journal of Econometrics, 160:235-245.

[3] Brooks, C. and Prokopczuk, M. (2011). The Dynamics of Commodity Prices. ICMA Centre Discussion Papers in Finance, DP2011-09.

[4] Chib, S. Nardari, F. and Shephard, N. (2002). Markov chain Monte Carlo methods for stochastic volatility models. Journal of Econometrics, 108:281316.

[5] Demarqui, F.N.(2010). "Uma classe mais flexível de modelos semiparamtricos para dados de sobrevivncia". UFMG. Doctoral Thesis. Belo Horizonte. URL: http://est.ufmg.br/portal/arquivos/doutorado/teses/TeseFabioDemarquiFinal.pdf

[6] Doornik, J.A.(2008). Ox Edit 5.10 [Computer Software]. Retrieved from http://www.doornik.com/ox/.

[7] Dowd, K.(2002). Measuring market risk. Wiley, England.

[8] Duffie, D. and Pan, J. (1997). An Overview of Value at Risk. The Journal of Derivatives, 3:7-49.

[9] Eraker, B. Johannes, M. and Polson, N. (2003). The Impact of Jumps in Volatility and Returns. The Journal of Finance, 58:1269-1299. 
[10] Gamerman, D. Santos, T. and Franco, G. (2013). A Non-Gaussian Family of State-Space Models With Exact Marginal Likelihood. Journal of Time Series Analysis, 34:625-645.

[11] Hendricks, D. (1995). Evaluation of Value-at-Risk Models Using Historical Data. Working paper, Federal Reserve Bank of New York.

[12] Ibrahim, J. G. Chen, M.H. and Sinha, D. (2001). Bayesian survival analysis. Springer-Verlag, New York.

[13] Kungl. Vetenskaps-Akademien. (2013). Understanding Asset Prices Scientific Background on the Sveriges Riksbank Prize in Economic Sciences in Memory of Alfred Nobel 2013, 1-55.

[14] Lee, S. and Maykland, P. (2008). Jumps in Financial Markets: A New Nonparametric Test and Jump Dynamics. The Review of Financial Studies, 21:2535-2563.

[15] Li, H. Wells M. and Yu, C. (2008). A Bayesian Analysis of Return Dynamics with Levy Jumps. Review of Financial Studies, 21:2345-2378.

[16] Merener, N. (2015). Concentrated Production and Conditional Heavy Tails in Commodity Returns. The Journal of Futures Markets, XX:1-20.

[17] Nakajima, J. and Omori, Y. (2007). Leverage, Heavy-Tails and Correlated Jumps in Stochastic Volatility Models. CARF Working Paper, CARF-F-107.

[18] Omori, Y. Chib, S. Shephard, N. and Nakajima, J. (2007). Stochastic volatility with leverage: Fast and efficient likelihood inference Journal of Econometrics, 140: 425-449.

[19] Prado, R. and West, M. (2010). Time Series Modeling, Computing and Inference. CRC Press, Boca Raton.

[20] Raftery, A.E. and Lewis, S.M. (1992). How many iterations in the Gibbs sampler?. Bayesian Statistics, 4:765-776.

[21] Riley, W. J. (2008). Algorithms for frequency jump detection. Metrologia, 45:S154-S161. 
[22] Schwartz, E. and Smith, J. (2000). Short-Term Variations and Long-Term Dynamics in Commodity Prices. Management Science, 46:893-911.

[23] The R foundation for Statistical Computing.(2015). R version 3.2.1 [Computer Software]. Retrieved from http://www.r-project.org/.

[24] Todorov, V. (2010). Variance Risk-Premium Dynamics: The Role of Jumps. Review of Financial Studies, 23:345-383.

[25] Triantafyllopoulos, K. (2008). Multivariate stochastic volatility with Bayesian dynamic linear models. Journal of Statistical Planning and Inference, 138:1021-1037.

[26] Warty, S. Lopes, H. and Polson, N. (2014). Sequential bayesian learning for stochastic volatility with variance-gamma jumps in returns. Insper Working Paper, WPE:340/2014.

[27] West, M. and Harrison, J. (1997). Bayesian Forecasting and Dynamic Models. Springer, New York.

[28] Wit, E. Heuvel, E and Romeijn, J. (2012). All models are wrong...: an introduction to model uncertainty. Statistica Neerlandica, DOI:10.1111. 


\section{Appendix A}

\section{Full Conditional Posterior Distributions for NGSV Model}

This appendix presents the conditional posterior distributions for the variables NGSVJ model. For simplifying notation, let $\Phi=\left(\theta_{t}, J_{t}^{y}, \gamma_{t}, \delta_{t}, \lambda_{t}, \sigma_{u}^{2}\right)$ be all other model variables, excluding the one which conditional posterior distribution is being calculated.

\section{A.1 Conditional Posterior Distribution for $\gamma_{t}$}

$$
\begin{aligned}
p\left(\gamma_{t}\right) & \sim \operatorname{Gamma}\left(\frac{\nu}{2}, \frac{\nu}{2}\right) \\
p\left(\gamma_{t} \mid y_{t}, \Phi_{[-\gamma]}\right) & \propto\left(\gamma_{t}\right)^{\frac{\nu}{2}-1} e^{-\gamma_{t} \frac{\nu}{2}}\left(\frac{1}{\gamma_{t}^{-1} \lambda_{t}^{-1}}\right)^{\frac{1}{2}} \exp \left(-\frac{\left(Y_{t}-F_{t} \theta_{t}-J_{t}\right)^{2}}{2 \gamma_{t}^{-1} \lambda_{t}^{-1}}\right) \\
p\left(\gamma_{t} \mid y_{t}, \Phi_{[-\gamma]}\right) & \propto\left(\gamma_{t}\right)^{\frac{\nu}{2}+\frac{1}{2}-1} \exp \left(-\gamma_{t}\left[\frac{\nu}{2}+\lambda_{t} \frac{\left(Y_{t}-F_{t} \theta_{t}-J_{t}\right)^{2}}{2}\right]\right) \\
p\left(\gamma_{t} \mid y_{t}, \Phi_{[-\gamma]}\right) & \sim \operatorname{Gamma}\left(\frac{\nu}{2}+\frac{1}{2}, \frac{\nu}{2}+\lambda_{t} \frac{\left(Y_{t}-F_{t} \theta_{t}-J_{t}\right)^{2}}{2}\right) .
\end{aligned}
$$




\section{A.2 Conditional Posterior Distribution for $\delta_{t}$}

$$
p\left(\delta_{t}\right) \sim \operatorname{Gamma}\left(\frac{\nu}{2}, \frac{\nu}{2}\right)
$$

$p\left(\delta_{t} \mid y_{t}, \Phi_{[-\delta]}\right) \propto\left(\delta_{t}\right)^{\frac{\nu}{2}-1} e^{-\delta_{t} \frac{\nu}{2}}\left|\delta_{t}^{-1}\left(\sigma_{\mu}^{2}\right)\right|^{-\frac{1}{2}} \exp \left(-\frac{\left(\theta_{t}-\theta_{t-1}\right)\left(\delta_{t}^{-1}\left(\sigma_{\mu}^{2}\right)\right)^{-1}\left(\theta_{t}-\theta_{t-1}\right)}{2}\right)$

$p\left(\delta_{t} \mid y_{t}, \Phi_{[-\delta]}\right) \propto\left(\delta_{t}\right)^{\frac{\nu}{2}+\frac{1}{2}-1} \exp \left(-\delta_{t}\left[\frac{\nu}{2}+\frac{\left(\theta_{t}-\theta_{t-1}\right)\left(\sigma_{\mu}^{2}\right)^{-1}\left(\theta_{t}-\theta_{t-1}\right)}{2}\right]\right)$

$p\left(\delta_{t} \mid y_{t}, \Phi_{[-\delta]}\right) \sim \operatorname{Gamma}\left(\frac{\nu}{2}+\frac{1}{2}, \frac{\nu}{2}+\frac{\left(\theta_{t}-\theta_{t-1}\right)\left(\sigma_{\mu}^{2}\right)^{-1}\left(\theta_{t}-\theta_{t-1}\right)}{2}\right)$.

\section{A.3 Conditional Posterior Distribution for $\sigma_{\mu}^{2}$}

$$
\begin{aligned}
p\left(\sigma_{\mu}^{2}\right) & \sim \text { InverseGamma }\left(a_{0}, b_{0}\right) \\
p\left(\sigma_{\mu}^{2} \mid y_{t}, \Phi_{\left[-\sigma_{\mu}^{2}\right]}\right) & \propto\left(\frac{1}{\sigma_{\mu}^{2}}\right)^{a_{0}+1} e^{-\frac{b_{0}}{\sigma_{\mu}^{2}}} \prod_{i=2}^{n}\left(\frac{1}{\delta_{i}^{-1} \sigma_{\mu}^{2}}\right)^{\frac{1}{2}} \exp \left(-\sum_{i=2}^{n} \frac{\left(\theta_{i}-\theta_{i-1}\right)^{2}}{2 \delta_{i}^{-1} \sigma_{\mu}^{2}}\right) \\
p\left(\sigma_{\mu}^{2} \mid y_{t}, \Phi_{\left[-\sigma_{\mu}^{2}\right]}\right) & \propto\left(\frac{1}{\sigma_{\mu}^{2}}\right)^{a_{0}+1} e^{-\frac{b_{0}}{\sigma_{\mu}^{2}}}\left(\frac{1}{\sigma_{\mu}^{2}}\right)^{\frac{n}{2}} \exp \left(-\frac{1}{2 \sigma_{\mu}^{2}} \sum_{i=2}^{n} \delta_{i}\left(\theta_{i}-\theta_{i-1}\right)^{2}\right) \\
p\left(\sigma_{\mu}^{2} \mid y_{t}, \Phi_{\left[-\sigma_{\mu}^{2}\right]}\right) & \propto\left(\sigma_{\mu}^{2}\right)^{-\left(a_{0}+\frac{n}{2}+1\right)} \exp \left(-\frac{1}{\sigma_{\mu}^{2}}\left[b_{0}+\frac{\sum_{i=2}^{t} \delta_{i}\left(\theta_{i}-\theta_{i-1}\right)^{2}}{2}\right]\right) \\
p\left(\sigma_{\mu}^{2} \mid y_{t}, \Phi_{\left[-\sigma_{\mu}^{2}\right]}\right) & \sim \text { InverseGamma }\left(a_{0}+\frac{n}{2}, b_{0}+\frac{\sum_{i=2}^{t} \delta_{i}\left(\theta_{i}-\theta_{i-1}\right)^{2}}{2}\right) .
\end{aligned}
$$




\section{Appendix B}

\section{Full Conditional Posterior Distributions for Jump Components}

This appendix presents the conditional posterior distributions for the jump components: $\mu_{y}, \sigma_{y}^{2}, \xi_{t+1}^{y} N_{t+1}^{y}$ and $\rho$. For simplifying notation, let $\Phi=\left(\theta_{t}, J_{t}^{y}, \gamma_{t}, \delta_{t}, \lambda_{t}, \sigma_{u}^{2}\right)$ be all other model variables, excluding the one which conditional posterior distribution is being calculated. 


\section{B.1 Conditional Posterior Distribution for $\mu_{y}$}

$$
\begin{aligned}
p\left(\mu_{y}\right) & \sim N(m, v) \\
p\left(\mu_{y} \mid y_{t}, \Phi_{\left[-\mu_{y}\right]}\right) & \propto \exp \left(-\frac{1}{2 v}\left(\mu_{y}-m\right)^{2}\right) \exp \left(-\frac{1}{2 \sigma_{y}^{2}} \sum_{\substack{i=1 \\
J_{i} \neq 0}}^{t}\left(\xi_{i+1}^{y}-\mu_{y}\right)^{2}\right) \\
p\left(\mu_{y} \mid y_{t}, \Phi_{\left[-\mu_{y}\right]}\right) & \propto \exp \left(-\frac{1}{2 v}\left(\mu_{y}-m\right)^{2}-\frac{1}{2 \sigma_{y}^{2}}\left(\sum_{\substack{i=1 \\
J_{i} \neq 0}}^{t}\left(\xi_{i+1}^{y}\right)^{2}-2 \mu_{y} n \overline{\xi^{y}}+n \mu_{y}^{2}\right)\right) \\
p\left(\mu_{y} \mid y_{t}, \Phi_{\left[-\mu_{y}\right]}\right) & \propto \exp \left(-\frac{1}{2 v \sigma_{y}^{2}}\left[\left(\sigma_{y}^{2}+n v\right) \mu_{y}^{2}-2 \mu_{y}\left(m \sigma_{y}^{2}+v n \overline{\xi^{y}}\right)\right]\right) \\
p\left(\mu_{y} \mid y_{t}, \Phi_{\left[-\mu_{y}\right]}\right) & \propto \exp \left(-\frac{1}{2 \frac{v \sigma_{y}^{2}}{\sigma_{y}^{2}+n v}}\left[\mu_{y}-\frac{m \sigma_{y}^{2}+v n \overline{\xi^{y}}}{\sigma_{y}^{2}+n v}\right]^{2}\right) \\
p\left(\mu_{y} \mid y_{t}, \Phi_{\left[-\mu_{y}\right]}\right) & \sim N\left(\frac{m \sigma_{y}^{2}+v n \overline{\xi^{y}}}{\sigma_{y}^{2}+n v}, \frac{v \sigma_{y}^{2}}{\sigma_{y}^{2}+n v}\right) .
\end{aligned}
$$

\section{B.2 Conditional Posterior Distribution for $\sigma_{y}^{2}$}

$$
\begin{aligned}
p\left(\sigma_{y}^{2}\right) & \sim \text { InverseGamma }(\alpha, \beta) \\
p\left(\sigma_{y}^{2} \mid y_{t}, \Phi_{\left[-\sigma_{y}^{2}\right]}\right) & \propto\left(\frac{1}{\sigma_{y}^{2}}\right)^{\alpha+1} e^{-\frac{\beta}{\sigma_{y}^{2}}}\left(\frac{1}{\sigma_{y}^{2}}\right)^{\frac{n}{2}} \exp \left(-\frac{1}{2 \sigma_{y}^{2}} \sum_{\substack{i=1 \\
J_{i} \neq 0}}^{t}\left(\xi_{i+1}^{y}-\mu_{y}\right)^{2}\right) \\
p\left(\sigma_{y}^{2} \mid y_{t}, \Phi_{\left[-\sigma_{y}^{2}\right]}\right) & \propto\left(\sigma_{y}^{2}\right)^{-\left(\alpha+\frac{n}{2}+1\right)} \exp \left(-\frac{1}{\sigma_{y}^{2}}\left[\beta+\frac{\sum_{\substack{i=1 \\
J_{i} \neq 0}}^{t}\left(\xi_{i+1}^{y}-\mu_{y}\right)^{2}}{2}\right]\right) \\
p\left(\sigma_{y}^{2} \mid y_{t}, \Phi_{\left[-\sigma_{y}^{2}\right]}\right) & \sim \text { InverseGamma }\left(\alpha+\frac{n}{2}, \beta+\frac{\sum_{\substack{i=1 \\
J_{i} \neq 0}}^{t}\left(\xi_{i+1}^{y}-\mu_{y}\right)^{2}}{2}\right) .
\end{aligned}
$$




\section{B.3 Conditional Posterior Distribution for $\xi_{t+1}^{y}$}

$$
\begin{aligned}
p\left(\xi_{t+1}^{y}\right) & \sim N\left(\mu_{y}, \sigma_{y}^{2}\right) \\
p\left(\xi_{t+1}^{y} \mid y_{t}, \Phi_{\left[-\xi^{y}\right]}\right) & \propto \exp \left(-\frac{1}{2 \sigma_{y}^{2}}\left(\xi_{t+1}^{y}-\mu_{y}\right)^{2}\right) \exp \left(-\frac{1}{2 \gamma_{t}^{-1} \lambda_{t}^{-1}}\left(y_{t}-F_{t} \theta_{t}-\xi_{t+1}^{y}\right)^{2}\right) \\
p\left(\xi_{t+1}^{y} \mid y_{t}, \Phi_{\left[-\xi^{y}\right]}\right) & \propto \exp \left(-\frac{1}{2}\left[\frac{\left(\xi_{t+1}^{y}\right)^{2}-2 \xi_{t+1}^{y} \mu_{y}}{\sigma_{y}^{2}}+\frac{\left.\left(\xi_{t+1}^{y}\right)^{2}+2 \xi_{t+1}^{y} F_{t} \theta_{t}-2 \xi_{t+1}^{y} y_{t}\right]}{\gamma_{t}^{-1} \lambda_{t}^{-1}}\right]\right) \\
p\left(\xi_{t+1}^{y} \mid y_{t}, \Phi_{\left[-\xi^{y}\right]}\right) & \propto \exp \left(-\frac{1}{2 \sigma_{y}^{2} \gamma_{t}^{-1} \lambda_{t}^{-1}}\left[\left(\sigma_{y}^{2}+\gamma_{t}^{-1} \lambda_{t}^{-1}\right)\left(\xi_{t+1}^{y}\right)^{2}-2 \xi_{t+1}^{y}\left(\mu_{y} \gamma_{t}^{-1} \lambda_{t}^{-1}+y_{t} \sigma_{y}^{2}-F_{t} \theta_{t} \sigma_{y}^{2}\right)\right]\right) \\
p\left(\xi_{t+1}^{y} \mid y_{t}, \Phi_{\left[-\xi^{y}\right]}\right) & \propto \exp \left(-\frac{1}{2 \frac{\sigma_{y}^{2} \gamma_{t}^{-1} \lambda_{t}^{-1}}{\sigma_{y}^{2} \gamma_{t}^{-1} \lambda_{t}^{-1}}}\left[\xi_{t+1}^{y}-\frac{\mu_{y} \gamma_{t}^{-1} \lambda_{t}^{-1}+y_{t} \sigma_{y}^{2}-F_{t} \theta_{t} \sigma_{y}^{2}}{\sigma_{y}^{2}+\gamma_{t}^{-1} \lambda_{t}^{-1}}\right]^{2}\right) \\
p\left(\xi_{t+1}^{y} \mid y_{t}, \Phi_{\left[-\xi^{y}\right]}\right) & \sim N\left(\frac{\mu_{y} \gamma_{t}^{-1} \lambda_{t}^{-1}+y_{t} \sigma_{y}^{2}-F_{t} \theta_{t} \sigma_{y}^{2}}{\sigma_{y}^{2}+\gamma_{t}^{-1} \lambda_{t}^{-1}}, \frac{\sigma_{y}^{2} \gamma_{t}^{-1} \lambda_{t}^{-1}}{\sigma_{y}^{2}+\gamma_{t}^{-1} \lambda_{t}^{-1}}\right) .
\end{aligned}
$$

\section{B.4 Conditional Posterior Distribution for $\rho$}

$$
\begin{aligned}
p(\rho) & \sim \operatorname{Beta}(\alpha, \beta) \\
p\left(\rho \mid Y_{n}, \Phi_{[-\rho]}\right) & \propto \rho^{\alpha-1}(1-\rho)^{\beta-1} \prod_{i=1}^{n} \rho^{N_{i}^{y}}(1-\rho)^{1-N_{i}^{y}} \\
p\left(\rho \mid Y_{n}, \Phi_{[-\rho]}\right) & \propto \rho^{\alpha-1}(1-\rho)^{\beta-1} \rho^{\sum_{i=0}^{n} N_{i}^{y}}(1-\rho)^{n-\sum_{i=0}^{n} N_{i}^{y}} \\
p\left(\rho \mid Y_{n}, \Phi_{[-\rho]}\right) & \propto \rho^{\alpha+\sum_{i=0}^{n} N_{i}^{y}-1}(1-\rho)^{\beta+n-\sum_{i=0}^{n} N_{i}^{y}-1} \\
p\left(\rho \mid Y_{n}, \Phi_{[-\rho]}\right) & \sim \operatorname{Beta}\left(\alpha+\sum_{i=0}^{n} N_{i}^{y}, \beta+n-\sum_{i=0}^{n} N_{i}^{y}\right)
\end{aligned}
$$

\section{B.5 Conditional Posterior Distribution for $N_{t+1}^{y}$}

$$
\begin{aligned}
& P\left(N_{t+1}^{y}=1 \mid, \Phi_{[-N y]}\right) \propto \rho P\left(Y_{t+1} \mid N_{t+1}^{y}=1, \Phi_{\left[-N^{y}\right]}\right) \\
& P\left(N_{t+1}^{y}=0 \mid, \Phi_{\left[-N^{y}\right]}\right) \propto(1-\rho) P\left(Y_{t+1} \mid N_{t+1}^{y}=0, \Phi_{\left[-N^{y}\right]}\right)
\end{aligned}
$$


Since $N^{y}$ can assume only two values, 0 or 1 :

$$
P\left(N_{t+1}^{y}=1 \mid, \Phi_{\left[-N^{y}\right]}\right)=\frac{\rho P\left(Y_{t+1} \mid N_{t+1}^{y}=1, \Phi_{\left[-N^{y}\right]}\right)}{\rho P\left(Y_{t+1} \mid N_{t+1}^{y}=1, \Phi_{\left[-N^{y}\right]}\right)+(1-\rho) P\left(Y_{t+1} \mid N_{t+1}^{y}=0, \Phi_{\left[-N^{y}\right]}\right)} .
$$

Using the idea proposed by Brooks and Prokopczuk (2011) [3], be $\alpha$ a threshold so that:

$$
N_{t+1}^{y}=\left\{\begin{array}{ll}
1 & \text { if } \quad P\left(N_{t+1}^{y}=1 \mid, \Phi_{\left[-N^{y}\right]}\right)>\alpha \\
0 & \text { if } \quad P\left(N_{t+1}^{y}=1 \mid \Phi\right) \leq \alpha
\end{array} .\right.
$$




\section{Appendix C}

\section{Sampling from $\lambda_{t}$}

This appendix shows how the posterior sample from $\lambda_{t}$ is obtained, as described by Gamerman et al. (2013) [10].

The joint distribution of $\left(\lambda \mid Y_{n}, \varphi\right)$ has density

$$
p\left(\lambda \mid \varphi, Y_{n}\right)=p\left(\lambda_{n} \mid \varphi, Y_{n}\right) \prod_{t=1}^{n-1} p\left(\lambda_{t} \mid \lambda_{t+1}, \varphi, Y_{t}\right) p\left(\varphi \mid Y_{n}\right)
$$

where the distribution of $\left(\lambda_{t} \mid \lambda_{t+1}, \varphi, Y_{t}\right)$ is given by

$$
\lambda_{t}-\omega \lambda_{t+1} \mid \lambda_{t+1}, \varphi, Y_{t} \sim \operatorname{Gamma}\left((1-\omega) a_{t}, b_{t}\right), \forall t \geq 0,
$$

where $a_{t}$ and $b_{t}$ are the filtering parameters. The on-line or updated distribution $\lambda_{t} \mid \varphi, Y_{t}$, where $\varphi=\left(\theta_{t}, J_{t}^{y}, \gamma_{t}, \omega\right)$, is given by:

$$
\begin{aligned}
p\left(\lambda_{t}\right) & \sim \operatorname{Gamma}\left(\omega a_{t-1}, \omega b_{t-1}\right) \\
p\left(\lambda_{t} \mid \varphi, Y_{t}\right) & \propto\left(\lambda_{t}\right)^{\omega a_{t-1}-1} e^{-\lambda_{t} \omega b_{t-1}}\left(\frac{1}{\gamma_{t}^{-1} \lambda_{t}^{-1}}\right)^{\frac{1}{2}} \exp \left(-\frac{\left(Y_{t}-F_{t} \theta_{t}-J_{t}\right)^{2}}{2 \gamma_{t}^{-1} \lambda_{t}^{-1}}\right) \\
p\left(\lambda_{t} \mid \varphi, Y_{t}\right) & \propto\left(\lambda_{t}\right)^{\omega a_{t-1}+\frac{1}{2}-1} \exp \left(-\lambda_{t}\left[\omega b_{t-1}+\gamma_{t} \frac{\left(Y_{t}-F_{t} \theta_{t}-J_{t}\right)^{2}}{2}\right]\right) \\
p\left(\lambda_{t} \mid \varphi, Y_{t}\right) & \sim \operatorname{Gamma}\left(\omega a_{t-1}+\frac{1}{2}, \omega b_{t-1}+\gamma_{t} \frac{\left(Y_{t}-F_{t} \theta_{t}-J_{t}\right)^{2}}{2}\right)
\end{aligned}
$$


Based on that Theorem and a sample of $p\left(\varphi \mid Y_{n}\right)$, an exact sample of the joint distribution $\left(\lambda \mid Y_{n}, \varphi\right)$ can be obtained following the algorithm:

1. set $\mathrm{t}=\mathrm{n}$ and sample $p\left(\lambda_{n} \mid \varphi, Y_{n}\right)$;

2. set $\mathrm{t}=\mathrm{t}-1$ and sample $p\left(\lambda_{t} \mid \lambda_{t+1}, \varphi, Y_{t}\right)$;

3. if $\mathrm{t}>1$, go back to step 2 ; otherwise, the sample of $\left(\lambda_{1}, \ldots, \lambda_{n} \mid \varphi, Y_{n}\right)$ is complete.

This Theorem allows the implementation of the algorithms step 2 and enables to obtain an exact sample from the smoothed distribution of the states conditioned on other parameters. 


\section{Appendix D}

\section{MCMC Chains for NGSVJ Model}

This appendix show MCMC results for static parameters for NGSVJ model application on S\&P 500 and iBovespa applications. 


\section{D.1 S\&P 500 MCMC results}

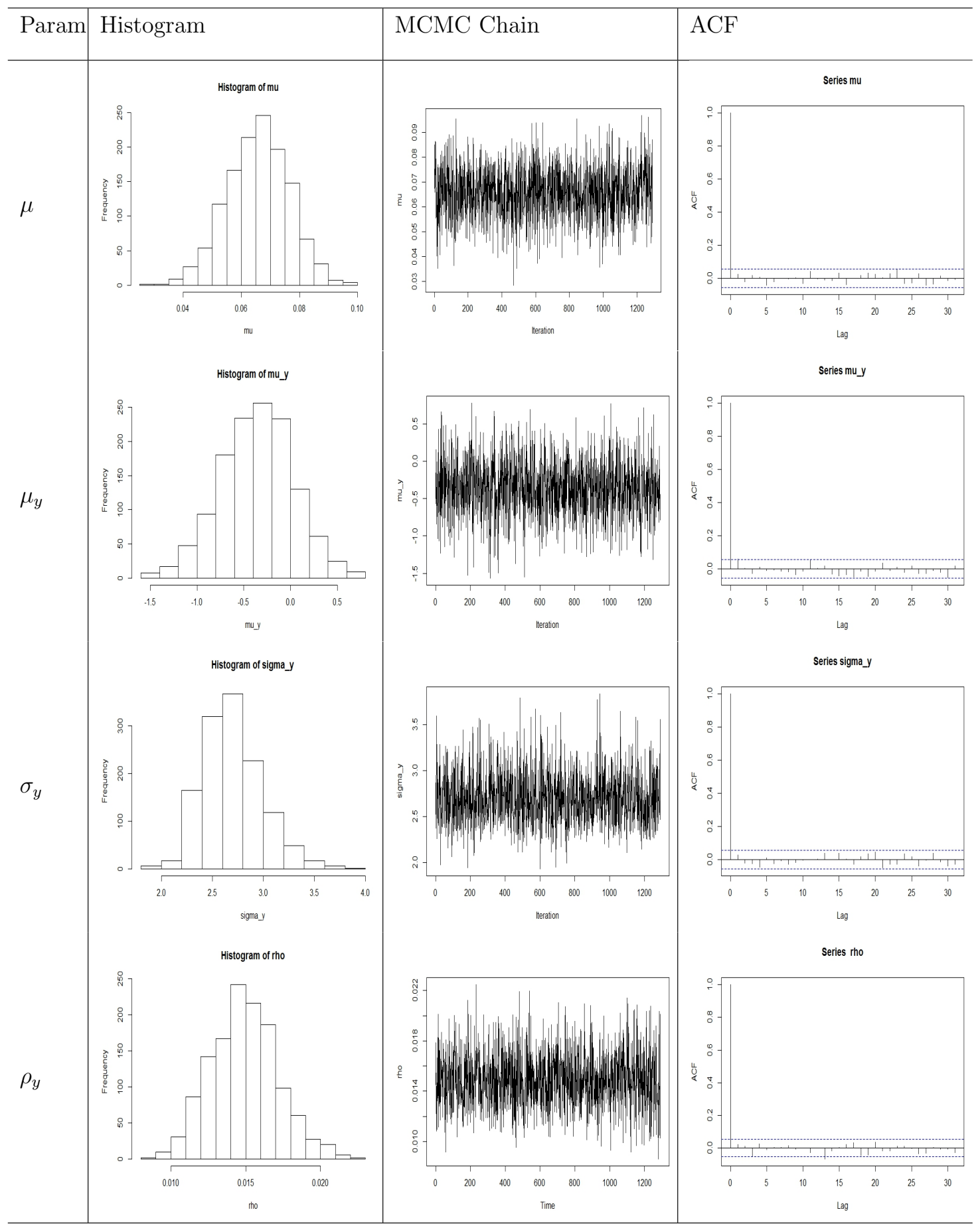

Figure D.1: MCMC results for S\&P 500 log returns data. 


\section{D.2 iBovespa MCMC results}

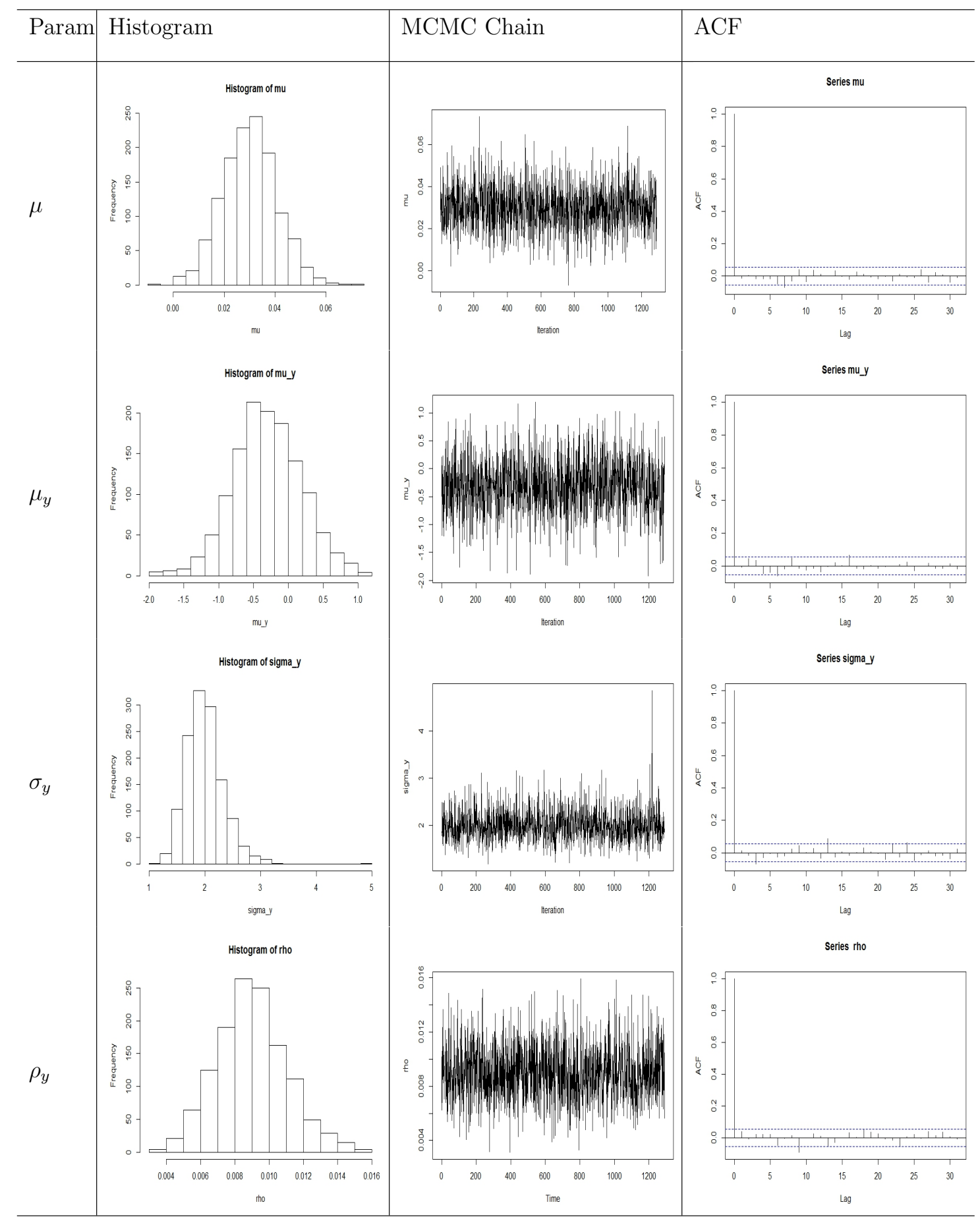

Figure D.2: MCMC results for iBovespa log returns data. 